\title{
Fujiki relations and fibrations of irreducible symplectic varieties
}

\author{
Martin Schwald
}

\begin{abstract}
This paper concerns different types of singular complex projective varieties generalizing irreducible symplectic manifolds. We deduce from known results that the generalized BeauvilleBogomolov form satisfies the Fujiki relations and has rank $\left(3,0, b_{2}(X)-3\right)$. This enables us to study fibrations of these varieties; imposing the newer definition from [GKP16, Definition 8.16.2] we show that they behave much like irreducible symplectic manifolds.
\end{abstract}

Keywords. irreducible symplectic, primitive symplectic, symplectic singularities, Lagrangian fibrations, Fujiki relation

2010 Mathematics Subject Classification. 14E99, 14D99, 14J99

[Français]

\section{Relations de Fujiki et fibrations de variétés symplectiques irréductibles}

Résumé. Cet article concerne différents types de variétés projectives complexes singulières qui généralisent les variétés symplectiques irréductibles. Nous déduisons de résultats connus que la forme quadratique de Beauville-Bogomolov généralisée vérifie les relations de Fujiki et a pour signature $\left(3,0, b_{2}(X)-3\right)$. Cela nous permet d'étudier les fibrations de ces variétés ; en imposant la récente définition 8.16.2 issue de [GKP16], nous montrons qu'elles se comportent de façon très similaire aux variétés symplectiques irréductibles lisses.

Received by the Editors on June 1st, 2018, and in final form on March 19, 2020.

Accepted on April 18, 2020.

Martin Schwald

Martin Schwald, Universität Duisburg-Essen, Fakultät für Mathematik, Thea-Leymann-Str. 9, 45127 Essen

e-mail: martin.schwald@uni-due.de

The author gratefully acknowledges support by the DFG-Graduiertenkolleg GK1821 "Cohomological Methods in Geometry" at the University of Freiburg and partial support by DFG-SFB/TRR 45.

(C) by the author(s)

This work is licensed under http://creativecommons.org/licenses/by-sa/4.0/ 


\section{Contents}

1. Introduction. . . . . . . . . . . . . . . . . . . . . . . . . . . . . . . . 2

2. Methods . . . . . . . . . . . . . . . . . . . . . . 5

3. Generalized Beauville-Bogomolov form . . . . . . . . . . . . . . . . . . . . . 9

4. Proofs of the main results . . . . . . . . . . . . . . . . . . . . . . . . . . 10

References. . . . . . . . . . . . . . . . . . . . . . . 17

\section{Introduction}

\subsection{Symplectic varieties}

Irreducible symplectic manifolds were introduced by Beauville [Bea83b, p. 763f] and play an important role in the structure theory of complex manifolds of Kodaira dimension zero as they occur as factors in the famous Beauville-Bogomolov decomposition theorem [Bea83b, th. 2]. A singular version of the decomposition theorem was recently proved [HP19, Theorem 1.5], building on the work of [GKP16, GGK19, DG18, Dru18] Remarkably, the class of singular symplectic factors emerging from this approach [GKP16, Definition 8.16.2], we call them here primitive symplectic varieties, differs from the types of singular symplectic varieties studied before.

To understand the geometry of primitive symplectic varieties better, we want to classify their fibrations. Our main results show that they behave much like irreducible symplectic manifolds and are arguably closer to them than other definitions. While we prove in Theorem 2 and 3 that many of the properties that are well known for irreducible symplectic manifolds hold for a bigger class of symplectic varieties, which we call here irreducible symplectic varieties, Theorem 4 holds for primitive symplectic varieties but not completely for irreducible symplectic varieties.

We briefly recall the relevant definitions required to state our main results. The different notions in the literature generalizing holomorphic symplectic manifolds to a singular setting agree in the existence of a symplectic form on the smooth locus, which is best seen as a reflexive 2 -form.

Reflexive differential forms are holomorphic $p$-forms on the smooth locus $X_{\text {reg }}$ of a normal variety $X$. We denote the associated sheaf on $X$ by $\Omega_{X}^{[p]}$ and get

$$
\Omega_{X}^{[p]} \cong i_{*} \Omega_{X_{\text {reg }}}^{p} \cong\left(\Omega_{X}^{p}\right)^{* *} \quad \text { and } \quad \mathrm{H}^{0}\left(X, \Omega_{X}^{[p]}\right) \cong \mathrm{H}^{0}\left(X_{\text {reg }}, \Omega_{X_{\text {reg }}}^{p}\right),
$$

where $i: X_{\text {reg }} \hookrightarrow X$ is the inclusion. These forms satisfy good pull back properties, see Section 2.1. We recommend [Har80] for a reference on reflexive sheaves and [GKKP11, I-III], [KP16, I] for more information on reflexive differential forms.

A quasi-étale morphism $\pi: X^{\prime} \rightarrow X$ is a finite surjective morphism between complex varieties that is étale outside of an analytic subset $Z \subset X^{\prime}$ with $\operatorname{codim} Z \geq 2$.

Definition 1 (Symplectic varieties). Let X be a normal, complex projective variety.

(1) $A$ symplectic form on $X$ is a reflexive form $\omega \in \mathrm{H}^{0}\left(X, \Omega_{X}^{[2]}\right)$ that is non-degenerate at every point $x \in X_{\text {reg }}$.

(2) The pair $(X, \omega)$ is called a symplectic variety if for every resolution of singularities $v: \widetilde{X} \rightarrow X$ there is a

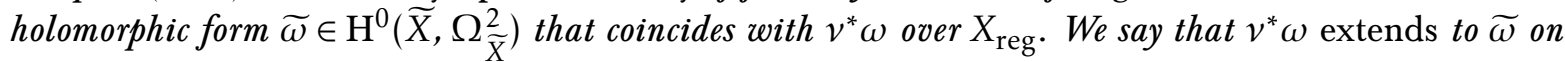
$\widetilde{X}$. 
(3) A symplectic variety $(X, \omega)$ is called irreducible symplectic if its irregularity $\mathrm{h}^{1}\left(X, \mathcal{O}_{X}\right)$ vanishes and if $\mathrm{h}^{0}\left(X, \Omega_{X}^{[2]}\right)=1$.

(4) We call an irreducible symplectic variety $(X, \omega)$ Namikawa symplectic if $X$ is $\mathbb{Q}$-factorial with $\operatorname{codim}_{\mathbb{C}} X_{\text {sing }} \geq 4$.

(5) We call a symplectic variety primitive symplectic if for all quasi-étale morphisms $\pi: X^{\prime} \rightarrow X$ with $X^{\prime}$ normal the reflexive ${ }^{1}$ pullback $\pi^{*} \omega \in \mathrm{H}^{0}\left(X^{\prime}, \Omega_{X^{\prime}}^{[2]}\right)$ generates the exterior algebra of reflexive forms on $X^{\prime}$, so

$$
\bigoplus_{p \in \mathbb{N}} \mathrm{H}^{0}\left(X^{\prime}, \Omega_{X^{\prime}}^{[p]}\right)=\mathbb{C}\left[\pi^{*} \omega\right] .
$$

Section 2.5 reviews properties of these notions and discusses their relations. Symplectic varieties were introduced by Beauville [Bea00, Definition 1.1].

Namikawa symplectic varieties, most prominently studied by Namikawa and Matsushita, have a wellbehaved deformation theory [Nam01c]. Primitive symplectic varieties are building blocks in a singular version of the Beauville-Bogomolov decomposition theorem. Using [GKP16, Proposition 6.9], we can consider irreducible symplectic varieties as a natural generalization of these two notions.

Irreducible symplectic manifolds are by definition simply connected, smooth irreducible symplectic varieties [Bea83b, p. 763f]. Using the Beauville-Bogomolov decomposition theorem we show that a smooth irreducible symplectic variety is always either simply connected or an étale quotient of an Abelian variety by a finite group of biholomorphic automorphisms, see Lemma 13. It is unknown if the latter case really occurs.

Irreducible symplectic manifolds are always primitive symplectic by [Bea83b, prop. 3]. In the singular case being primitive symplectic is known to be more restrictive, see Example 26. This is our motivation to study how their geometric properties differ.

\subsection{Main results}

On an irreducible symplectic variety $X$, Namikawa and Kirschner constructed in [Nam01c, Theorem 8 (2)], [Kir15, Definition 3.2.7] an important quadratic form $q_{X}$ on $\mathrm{H}^{2}(X, \mathbb{C})$, the Beauville-Bogomolov form. Its definition will be recalled in Section 3. We first prove the following minor generalization of results of Matsushita [Mat15, Proposition 4.1], [Mat01, Theorem 1.2].

Theorem 2 (Fujiki relations, index of the Beauville-Bogomolov form). Let $(X, \omega)$ be a 2n-dimensional, irreducible symplectic variety. The Beauville-Bogomolov form $q_{X}$ has the following properties:

(1) There is a number $c_{X} \in \mathbb{R}^{+}$, called Fujiki constant, such that for all $\alpha \in \mathrm{H}^{2}(X, \mathbb{C})$ the following, so-called Fujiki relation, holds, ${ }^{2}$

$$
c_{X} \cdot q_{X}(\alpha)^{n}=\int_{X} \alpha^{2 n}
$$

In particular for classes of Cartier divisors $d=c_{1}\left(\mathcal{O}_{X}(D)\right)$, this relates the Beauville-Bogomolov form to the intersection product via $c_{X} \cdot q_{X}(d)^{n}=D^{2 n}$.

(2) The restriction of $q_{X}$ to $\mathrm{H}^{2}(X, \mathbb{R}) \rightarrow \mathbb{R}$ is a real quadratic form of index $\left(3,0, b_{2}(X)-3\right)$.

Part (2) implies the existence of a $q_{X}$-orthogonal decomposition $\mathrm{H}^{2}(X, \mathbb{R})=V_{+} \oplus V_{-}$with $\operatorname{dim}_{\mathbb{R}} V_{+}=3$, such that $q_{X}$ is positive definite on $V_{+}$and negative definite on $V_{-}$, see Proposition 25.

In the smooth case, these properties were proved by Fujiki and Beauville [Fuj87, Theorem 4.7], [Bea83b, th. 5(a)]. Matsushita generalized them to Namikawa symplectic varieties [Mat01, Theorem 1.2], [Mat15, Proposition 4.1].

Matsushita uses his versions of Theorem 2 to show that fibrations of irreducible symplectic manifolds and Namikawa symplectic varieties have very special properties, [Mat99, Theorem 2], [Mat00, Corollary 1], [Mat01, Corollary 1.4], [Mat15, Theorem 1.2, 1.10]. As our main results, the following Theorems 3 and 4, we

\footnotetext{
${ }^{1}$ This reflexive pullback exists e.g. due to [Har80, Proposition 1.6]

${ }^{2}$ We will recall integration on singular cohomology in Section 2.3.
} 
prove that all these results hold for primitive symplectic varieties and we show, which of them are also true for all irreducible symplectic varieties.

Theorem 3 (Fibrations of irreducible symplectic varieties). Let $(X, \omega)$ be an irreducible symplectic variety of complex dimension $2 n$, together with a surjective morphism $f: X \rightarrow B$ with connected fibers onto a normal, projective variety $B$ with $0<\operatorname{dim} B<2 n$. Then the following properties hold.

(1) The base variety $B$ is an $n$-dimensional, $\mathbb{Q}$-factorial klt variety with Picard number $\rho(B)=1$.

(2) The singular locus of $X$ is mapped to a proper closed subset of B. In particular, the general fiber is smooth and entirely contained in $X_{\text {reg. }}$.

(3) The general fiber is an $n$-dimensional Abelian variety.

(4) Every fiber component of $f$ is an n-dimensional Lagrangian subvariety ${ }^{3}$ of $X$ and does not lie completely in $X_{\text {sing. }}$.

Theorem 4 (Fibrations of primitive symplectic varieties). Let $f: X \rightarrow B$ be as in Theorem 3. If $X$ is a primitive symplectic variety, then the following holds.

(1) The base variety $B$ is Fano, so $-K_{B}$ is ample.

(2) If $B$ is smooth, then $B \cong \mathbb{P}^{n}$.

Both parts of Theorem 4 were known to hold for all irreducible symplectic manifolds [Mat01, Theorem 2 (3)], [Hwa08, Theorem 1.2], [GL14, Theorem 1.1]. It is remarkable that if $X$ is an irreducible symplectic variety, then $K_{B}$ can vanish, hence part 1 can fail for irreducible symplectic varieties, as Example 26 shows.

To prove the results on fibrations we work as much as possible on the singular variety $X$. We hope that this will also allow to tackle further questions on Lagrangian fibrations. Open problems are for example a classification of the singular fibers and of the possible base varieties $B$. We still know little about its possible singularities. When $X$ is smooth, it is conjectured that $B$ is also smooth, but so far this got only proved for $\operatorname{dim} X \leq 4$ by Huybrechts-Xu and Ou [HX19], [Ou16, Theorem 1.2]. In general $B$ can certainly be singular, even if $X$ is primitive symplectic, [Mat15, Theorem 1.9].

\subsection{Outline of the paper}

We present in 2.1-2.4 our most important methods to prove our main theorems. They are the general extension theorem for differential forms from [GKKP11, Theorem 1.4], the existence of terminal models from [BCHM10, Corollary 1.4.3] and results on the Hodge theory of klt varieties that we explain in detail in [Schl7]. In particular we will need the Hodge decomposition of $\mathrm{H}^{2}(X, \mathbb{C})$ and a singular version of the Hodge-Riemann bilinear relations.

After recapitulating well-known properties of symplectic varieties in 2.5 , we prove that terminal models of irreducible or primitive symplectic varieties are Namikawa or primitive symplectic, respectively. In 2.6 we prove in Theorem 15 that singular fibers of Lagrangian fibrations are automatically also Lagrangian, implying that Lagrangian fibrations are always equidimensional. This requires a careful revision of the definitions of Lagrangian subvarieties and a subtle inductive argument. Theorem 15 will be used to show that the general fiber of a Lagrangian fibration is an Abelian variety, which in fact holds for fibrations of every symplectic variety. Here we make use of a recently proven special case of the Lipman-Zariski conjecture, which makes the proof even easier and more geometric than Matsushita's proof of [Mat01, Corollary 1.4 (2)]. We explain in Section 3 how the Beauville-Bogomolov form $q_{X}$ is defined in the singular setting in a way that is most suitable for direct computations in $\mathrm{H}^{2}(X, \mathbb{C})$.

In Section 4 we finally prove our main results. We deduce the Fujiki relation directly from a terminal model, and use our Hodge theoretic methods to calculate the index of $q_{X}$ by hand similarly like Matsushita. Theorem 2 is essential to prove the rest of Theorem 3 in the spirit of Matsushita's proof of [Mat01, Corollary 1.4]. Proving that $B$ is $\mathbb{Q}$-factorial becomes particularly involved because handling Weil divisors is much harder in the singular case. The proof of Theorem 4 uses the branched covering trick and going over to a terminal model, which we have seen to be primitive symplectic again.

\footnotetext{
${ }^{3}$ We discuss the definition of Lagrangian subvarieties in Section 2.6 .
} 


\subsection{Conventions}

We use the terminology of [Har77], in particular varieties are defined to be irreducible. We denote the smooth and the singular locus of a variety $X$ by $X_{\text {reg }}$ and $X_{\text {sing, }}$, respectively. For the terminology of the Minimal Model Program we refer to [KM98], but we use the sloppy term klt variety for a variety $X$ that carries an effective Weil divisor $D$, such that the pair $(X, D)$ is klt according to [KM98, Definition 2.34].

\subsection{Acknowledgment}

The results of this paper are an improved version of results of my $\mathrm{PhD}$ thesis, answering also Questions 6, 8 and Conjecture 7 that were stated in the introduction of the thesis, [Schl7]. I want to thank my supervisor S. Kebekus, as well as K. Oguiso, D. Greb, P. Graf, T. Kirschner and B. Taji for their advice and fruitful discussions. Special thanks to the anonymous referee for several corrections and improvements.

\section{Methods}

We recall here the extension theorem, terminal models, integration over singular cohomology classes and results on the Hodge theory for klt varieties. We recapitulate well-known properties of symplectic varieties that will imply that terminal models of irreducible or primitive symplectic varieties are Namikawa or primitive symplectic, respectively. Then we discuss the notions of Lagrangian subvarieties and fibrations and examine their singular fibers.

\subsection{Extension Theorem}

The extension theorem for differential forms [GKKP11, Theorem 1.4] shows that on a klt variety $X$ the pullback of every reflexive form $\alpha \in \mathrm{H}^{0}\left(X, \Omega_{X}^{[p]}\right)$ to a resolution of singularities can be extended over the exceptional locus. This allows us to construct pullbacks of reflexive forms along more general morphisms.

Theorem 5 (Pullbacks of reflexive forms, [Keb13, Theorem 1.3]). Let $f: Y \rightarrow X$ be a morphism between normal, complex quasi-projective varieties where $X$ is a klt variety. Then for every $p$ there is a natural pullback morphism $f^{*} \Omega_{X}^{[p]} \rightarrow \Omega_{Y}^{[p]}$, consistent with the natural pullback of Kähler differentials on $X_{\mathrm{reg}}$.

The consistency with the natural pullback of Kähler differentials is made precise in [Keb13, Theorem 5.2]. We will only consider the case when $f: Y \rightarrow X$ is surjective. Then this consistency means that the pullback morphism $f^{*} \Omega_{X}^{[p]} \rightarrow \Omega_{Y}^{[p]}$ agrees with the usual pullback of Kähler differentials wherever $X$ and $Y$ are smooth. In other words, for every $\alpha \in \mathrm{H}^{0}\left(X, \Omega_{X}^{[p]}\right)$ there is an $\widetilde{\alpha} \in \mathrm{H}^{0}\left(Y, \Omega_{Y}^{[p]}\right)$ that restricts on $Y_{\text {reg }} \cap f^{-1}\left(X_{\text {reg }}\right)$ to the natural pullback $f^{*}\left(\left.\alpha\right|_{X_{\text {reg }}}\right)$ of Kähler differentials. We call $\widetilde{\alpha}$ an extension of $f^{*} \alpha$ to $Y$. Pulling back reflexive forms is contravariant functorial with respect to $f$.

\subsection{Terminal models}

While not every singular variety admits a crepant resolution, complex projective klt varieties have always a terminal model. For canonical singularities a terminal model is a crepant partial resolution. This is an easy special case of [BCHM10, Corollary 1.4.3].

Theorem 6 (Terminal models, [BCHM10, page 413]). Let X be a complex projective variety with canonical singularities. Then every resolution $v: \widetilde{X} \rightarrow X$ of $X$ factors as $\widetilde{X} \stackrel{\widetilde{\pi}}{\longrightarrow} Y \stackrel{\pi}{\longrightarrow} X$, where $\widetilde{\pi}, \pi$ are birational and $Y$ is $a \mathbb{Q}$-factorial variety with at most terminal singularities, such that $\pi^{*} K_{X}=K_{Y}$. We call $Y$ a terminal model of $X$.

\subsection{Integration of singular top classes}

Recall that we integrate a top singular cohomology class $\phi \in \mathrm{H}^{2 n}(X, \mathbb{C})$ over an $n$-dimensional, compact, complex variety $X$ as $\int_{X} \phi:=[X] \cap \phi$. Here $[X] \in \mathrm{H}_{2 n}(X, \mathbb{Z})$ denotes the canonical fundamental class of $X$, 
induced by the complex structure on its smooth locus $X_{\text {reg }}$, [Sch17, Section 2.5]. This integration commutes with pullbacks by bimeromorphic maps.

Lemma 7 (Pullbacks of integrals, [Sch17, Lemma 22]). If $f: X \rightarrow Y$ is a bimeromorphic morphism of compact, complex varieties, then $\int_{Y} \alpha=\int_{X} f^{*} \alpha$ for all $\alpha \in \mathrm{H}^{2 n}(Y, \mathbb{C})$.

\subsection{Hodge Theory for klt varieties}

When $X$ is a compact complex variety with rational singularities, Namikawa noted in [Nam06, p. 143] that for every resolution of singularities $v: \widetilde{X} \rightarrow X$ the pullback morphism $v^{*}: \mathrm{H}^{2}(X, \mathbb{C}) \rightarrow \mathrm{H}^{2}(\widetilde{X}, \mathbb{C})$ is an embedding and therefore the Hodge structure on $\mathrm{H}^{2}(X, \mathbb{C})$ is pure of weight two. By similar arguments like in [Kal06, Lemma 2.7] it can be seen that for every reflexive 2-form $\alpha \in \mathrm{H}^{0}\left(X, \Omega_{X}^{[2]}\right)$ for which $f^{*} \alpha$ extends to a form $\widetilde{\alpha} \in \mathrm{H}^{0}\left(\widetilde{X}, \Omega_{X}^{2}\right)$ there is a class $\underline{\alpha} \in \mathrm{H}^{2}(X, \mathbb{C})$ with $f^{*} \underline{\alpha}=[\widetilde{\alpha}] \in \mathrm{H}^{2}(\widetilde{X}, \mathbb{C})$. When we have an extension theorem for reflexive forms on $X$ like Theorem 5, it follows that $\alpha \mapsto \underline{\alpha}$ defines an isomorphism $\mathrm{H}^{0}\left(X, \Omega_{X}^{[2]}\right) \cong \mathrm{H}^{2,0}(X)$. The author worked this out and drew the following consequences in his thesis.

Theorem 8 (Hodge decomposition of $\mathrm{H}^{2}(X, \mathbb{C}$ ), [Sch17, Theorem 37], $c f$. also [Kir15, B.2.7-B.2.9], [GKP16, Proposition 6.9]). When $X$ is a complex projective klt variety, then we have $\mathrm{H}^{2,0}(X) \cong \mathrm{H}^{0}\left(X, \Omega_{X}^{[2]}\right)$ and $\mathrm{H}^{0,2}(X) \cong \mathrm{H}^{2}\left(X, \mathcal{O}_{X}\right)$. For every resolution $v: \widetilde{X} \rightarrow X$ the pullback morphism $v^{*}: \mathrm{H}^{a, b}(X) \rightarrow \mathrm{H}^{a, b}(\widetilde{X})$ is bijective for $(a, b)=(2,0)$ or $(0,2)$ and injective for $(a, b)=(1,1)$.

Remark 9. Let $X$ be a complex projective klt variety. Every reflexive two-form $\alpha$ on $X$ defines by Theorem 8 a unique cohomology class in $\mathrm{H}^{2}(X, \mathbb{C})$ that we denote by $\underline{\alpha}$. When $f: Y \rightarrow X$ is a morphism of complex projective klt varieties and $\widetilde{\alpha}$ the extension of $f^{*} \alpha$ to $Y$ by Theorem 5 , then we have $\underline{\widetilde{\alpha}}=f^{*} \underline{\alpha}$ in $\mathrm{H}^{2}(Y, \mathbb{C})$ because pulling back is a morphism of Hodge structures.

Corollary 10 (Bilinear relations on klt varieties, [Sch17, Corollary 42]). Let X be an n-dimensional, complex projective klt variety. Every ample class $a \in \mathrm{H}^{2}(X, \mathbb{R})$ induces a sesquilinear form $\psi_{X, a}$ on $\mathrm{H}^{k}(X, \mathbb{C})$ defined by

$$
(v, w) \mapsto(-1)^{\frac{k(k-1)}{2}} \cdot \int_{X} v \cup \bar{w} \cup a^{n-k} .
$$

The so-called Hodge-Riemann bilinear relation $i^{p-q} \cdot \psi_{X, a}(v, v)>0$ holds for any non-zero $v$ with $v \cup a^{n-k+1}=0$ that can be written as the cup product of classes $v_{1}, \ldots, v_{r} \in \mathrm{H}^{2}(X, \mathbb{C})$.

We write $p:=\sum p_{j}, q:=\sum q_{j}$ and $k:=p+q$ where $\left(p_{j}, q_{j}\right)$ are such that the $\alpha_{j}$ lie in the $\mathrm{H}^{p_{j}, q_{j}}(X)$-part of the Hodge decomposition of $\mathrm{H}^{2}(X, \mathbb{C})$.

\subsection{Properties of symplectic varieties}

A symplectic variety has trivial canonical class, as the top exterior power of the symplectic form trivializes the canonical sheaf. It follows that symplectic varieties have canonical singularities [Bea00, Proposition 1.3], [KM98, Corollary 5.24]. Furthermore, Namikawa showed that a symplectic variety has at most terminal singularities if and only if its singular locus is at least of codimension four, [Nam01a, Corollary 1].

We point out that Matsushita uses in [Mat01] the property $\mathrm{h}^{2}\left(X, \mathcal{O}_{X}\right)=1$ of irreducible symplectic varieties instead of $h^{0}\left(X, \Omega_{X}^{[2]}\right)=1$. It follows from Theorem 8 that these two conditions are equivalent by the Hodge symmetry.

Proposition 11. Every terminal model $\pi: Y \rightarrow X$ of an irreducible symplectic variety $(X, \omega)$ is a Namikawa symplectic variety with the extension $\omega^{\prime} \in \mathrm{H}^{0}\left(Y, \Omega_{Y}^{[2]}\right)$ of $\pi^{*} \omega$ to $Y$ from Theorem 5.

Proof. Namikawa proved that $Y$ is again symplectic [Nam06, Remark 1]. Let $\widetilde{\pi}: \widetilde{X} \rightarrow Y$ be a resolution of $Y$. Then $\mathrm{h}^{1}\left(Y, \mathcal{O}_{Y}\right)=\mathrm{h}^{1}\left(\widetilde{X}, \mathcal{O}_{\widetilde{X}}\right)=\mathrm{h}^{1}\left(X, \mathcal{O}_{X}\right)=0$ as the irregularity is a birational invariant for varieties with rational singularities and $h^{0}\left(Y, \Omega_{Y}^{[2]}\right)=h^{0}\left(X, \Omega_{X}^{[2]}\right)=1$ by Theorem 5. Hence $(Y, \widetilde{\omega})$ is Namikawa symplectic by [Nam01a, Corollary 1]. 
Proposition 12. Every terminal model $\pi: Y \rightarrow X$ of a primitive symplectic variety $(X, \omega)$ is primitive symplectic with the extension $\omega^{\prime} \in \mathrm{H}^{0}\left(Y, \Omega_{Y}^{[2]}\right)$ of $\pi^{*} \omega$ to $Y$.

Proof. Let $g: Y^{\prime} \rightarrow Y$ be quasi-étale with $Y^{\prime}$ normal. Then the Stein factorization of $\pi \circ g$, [Har77, Corollary III.11.5], gives a morphism $\pi^{\prime}: Y^{\prime} \rightarrow X^{\prime}$ with connected fibers and a finite morphism $h: X^{\prime} \rightarrow X$ with $\pi \circ g=h \circ \pi^{\prime}$. Hence $\pi^{\prime}$ is birational because $\pi \circ g$ is generically finite. If $g$ is étale outside of $V \subset Y^{\prime}$, then $h$ is étale outside of $\pi^{\prime}(V)$ because $\pi$ and $\pi^{\prime}$ induce isomorphisms on the function fields. Thus $h$ is a quasi-étale. We note that normal, quasi-étale covers of klt varieties are also klt varieties by [KM98, Proposition 5.20]. A resolution $\widetilde{X}$ of $X^{\prime}$ is also a resolution of $Y^{\prime}$ and Theorem 5 implies $\mathrm{H}^{0}\left(Y^{\prime}, \Omega_{Y^{\prime}}^{[p]} \cong \mathrm{H}^{0}\left(\widetilde{X}, \Omega_{\widetilde{X}}^{p}\right) \cong \mathrm{H}^{0}\left(X^{\prime}, \Omega_{X^{\prime}}^{[p]}\right)\right.$ for all $p$. As $X$ is primitive symplectic and pulling back reflexive forms is functorial, the reflexive forms on $Y^{\prime}$ get generated by $\left(\pi^{\prime}\right)^{*} h^{*} \omega=g^{*} \omega^{\prime}$.

While we cannot prove that smooth irreducible symplectic varieties are irreducible symplectic manifolds as they were defined by Beauville, we can prove the following Lemma, which is related to [HNW11, Proposition A.1].

Lemma 13. Let $(X, \omega)$ be a smooth, irreducible symplectic variety in the sense of Definition 1. Then $X$ is either simply connected or an étale quotient of an Abelian variety by a finite group of biholomorphic automorphisms.

Proof. By the Beauville-Bogomolov decomposition theorem, there is a finite étale covering $\pi: \hat{X} \rightarrow X$ that splits as a product $\hat{X} \cong T \times X^{\prime} \times Y$, where $T$ is a complex torus, $X^{\prime}:=\prod_{i=1}^{k} X_{i}$ is the product of irreducible symplectic manifolds $X_{i}$, and $Y$ a product of at least three-dimensional Calabi-Yau manifolds. By going over to a finite étale covering of $\hat{X}$ we may assume $\pi$ to be a Galois covering, so $X \cong \hat{X} / G$ for $G \subset$ Aut $\hat{X}$ a group of biholomorphic automorphisms of $\hat{X}$ with $|G|=\operatorname{deg} \pi$.

We can identify $\mathrm{H}^{0}\left(X, \Omega_{X}^{2}\right)$ with the space of $G$-invariant holomorphic 2-forms $\mathrm{H}^{2}\left(\hat{X}, \Omega_{\hat{X}}^{2}\right)^{G}$, hence the latter one is generated by the pullback $\pi^{*} \omega$ of the symplectic form. As

$$
\mathrm{h}^{0}\left(X^{\prime}, \Omega_{X^{\prime}}^{1}\right)=\mathrm{h}^{0}\left(Y, \Omega_{Y}^{1}\right)=\mathrm{h}^{0}\left(Y, \Omega_{Y}^{2}\right)=0,
$$

we get by the Künneth formula a decomposition $\pi^{*} \omega=\pi_{T}^{*} \eta+\pi_{X^{\prime}}^{*} \omega^{\prime}$, where $\pi_{T}: \hat{X} \rightarrow T$ and $\pi_{X^{\prime}}: \hat{X} \rightarrow X^{\prime}$ are the projections, and $\eta \in \mathrm{H}^{0}\left(T, \Omega_{T}^{2}\right), \omega^{\prime} \in \mathrm{H}^{0}\left(X^{\prime}, \Omega_{X^{\prime}}^{2}\right)$ are 2 -forms on the factors. As $\pi$ is étale, $\pi^{*} \omega$ is also non-degenerate, hence $Y$ is trivial and the forms $\eta$ and $\omega^{\prime}$ are non-degenerate as well.

For every $f \in \operatorname{Aut}(\hat{X})$ there are automorphisms $g \in \operatorname{Aut}(T)$ and $h \in \operatorname{Aut}\left(X^{\prime}\right)$ such that $f=(g, h)$ [Bea83a, p. 8, Lemma], so $\pi_{T}^{*} \eta$ and $\pi_{X^{\prime}}^{*} \omega^{\prime}$ are $G$-invariant holomorphic 2-forms on $\hat{X}$. $\operatorname{As~}^{0}\left(X, \Omega_{X}^{2}\right)=1$, it follows that either $T$ or $X^{\prime}$ has to be trivial. If $X^{\prime}$ is trivial then $X \cong T / G$ and the proof is complete. ${ }^{4}$

Hence we can assume from now on that $T$ is trivial, so $X \cong X^{\prime} / G$. In this case, we show that every automorphism $f \in \operatorname{Aut}\left(X^{\prime}\right)$ has a fixed point. For this, let $\omega_{i}$ be the pullbacks of the symplectic forms of the factors $X_{i}$ to $X^{\prime}$. By [Bea83b, p. 762f, prop. 3-4] and the Künneth formula, $\bigoplus_{p \in \mathbb{N}} \mathrm{H}^{0}\left(X^{\prime}, \Omega_{X^{\prime}}^{p}\right)$ is generated by the wedge products of the $\omega_{i}$. In particular $\mathrm{H}^{j, 0}\left(X^{\prime}\right)=0$ for all odd $j$. As $(X, \omega)$ is symplectic, $f^{*}$ preserves a symplectic form on $X^{\prime}$. By rescaling the $\omega_{i}$ we may assume it to be the sum of the $\omega_{i}$.

By [Bea83a, p. 10, b)+c)] the automorphism $f$ acts on $X^{\prime}$ by first possibly permuting isomorphic factors $X_{i}$ and then applying automorphisms $f_{i} \in \operatorname{Aut}\left(X_{i}\right)$ on each factor. In particular there is a permutation $\sigma \in \mathcal{S}_{k}$ such that for all $i$ we have that $f^{*} \omega_{i}$ is a multiple of $\omega_{\sigma(i)}$. As $f^{*}$ preserves the sum of the $\omega_{i}$, it simply permutes the $\omega_{i}$, as well as their wedge products. Therefore $\operatorname{tr}\left(\left.f^{*}\right|_{H^{j, 0}\left(X^{\prime}\right)}\right)$ is zero for all odd $j$, non-negative for all even $j$ and it equals one for $j=0,2 \operatorname{dim}(X)$. Thus $f$ has a fixed point by the holomorphic Lefschetz fixed point formula, [GH94, p. 426]. As we assumed $X$ to be smooth, it follows that $G$ acts trivially on $X^{\prime}$, so $X \cong X^{\prime}$ is simply connected.

\subsection{Lagrangian fibrations}

We show in Theorem 17 that Lagrangian fibrations are equidimensional because also their singular fibers consist of Lagrangian subvarieties. A straightforward generalization of Matsushita's proof of [Mat00, Corollary 1], see Proposition 16, works for all fiber components that do not completely lie in the singular

\footnotetext{
${ }^{4}$ It is unclear if this case can occur. The argument in [HNW11, Proposition A.1] to exclude the torus factor is incomplete.
} 
locus. To complete the proof of Theorem 17 we use an additional inductive argument to make sure that there are no fiber components in $X_{\text {sing }}$.

Definition 14 (Lagrangian subvariety and fibration). Let $(X, \omega)$ be a symplectic variety and $F \subset X$ a subvariety with $F \not \subset X_{\text {sing }}$ and $\operatorname{dim} F=\frac{1}{2} \operatorname{dim} X$. If on every embedded ${ }^{5}$ resolution of singularities $v: \widetilde{X} \rightarrow X$ of $(X, F)$ the extension $\widetilde{\omega}$ of $v^{*} \omega$ vanishes on the strict transform of $F$, then we call $F$ a Lagrangian subvariety of $X$. $A$ Lagrangian fibration of $X$ is a surjective morphism $f: X \rightarrow B$ with connected fibers onto a normal, complex projective variety $B$, such that the general fiber is a Lagrangian subvariety of $X$.

The notion of Lagrangian subvarieties does not depend on the chosen embedded resolution because for two resolutions we can go over to a common resolution. Our definition is consistent with Matsushita's definition, [Mat00, Definition 1], [Mat05, Definition 1.2]. The following criteria can be used to test if a subvariety is Lagrangian.

Lemma 15 (Criteria of Lagrangian subvarieties). Let $F$ be a subvariety of a symplectic variety $(X, \omega)$ with $F \not \subset X_{\text {sing }}$ and $\operatorname{dim} F \geq \frac{1}{2} \operatorname{dim} X$. Then the following assertions hold.

(1) $F$ is Lagrangian if and only if $\left.\omega\right|_{X_{\mathrm{reg}} \cap F_{\mathrm{reg}}}$ vanishes as a Kähler differential on $X_{\mathrm{reg}} \cap F_{\mathrm{reg}}$.

(2) $F$ is Lagrangian if $i^{*} \underline{\omega}=0 \in \mathrm{H}^{2}(F, \mathbb{C})$ for the inclusion $i: F \hookrightarrow X$. The converse holds if $F$ is a klt variety.

Proof of (1). Let $v: \widetilde{X} \rightarrow X$ be an embedded resolution of $(X, F)$ with strict transform $\widetilde{i}: \widetilde{F} \hookrightarrow \widetilde{X}$ of $F$ and extension $\widetilde{\omega}$ of $v^{*} \omega$ to $\widetilde{X}$. As $v$ is an isomorphism over a dense open subset, the assumption $F \not \subset X_{\text {sing }}$ implies that the conditions $\left.\omega\right|_{X_{\text {reg }} \cap F_{\text {reg }}}=0$ and $\left.\widetilde{\omega}\right|_{\widetilde{F}}=0$ are equivalent. Moreover, linear algebra shows that both latter conditions imply $\operatorname{dim} F \leq \frac{1}{2} \operatorname{dim} X$ because $\omega$ is non-degenerate on $X_{\text {reg }}$, [Nam01b, Lemma 1.1]. Together with the assumption $\operatorname{dim} F \geq \frac{1}{2} \operatorname{dim} X$ this implies that the conditions $\left.\omega\right|_{X_{\text {reg }} \cap F_{\text {reg }}}=0$ and $\left.\widetilde{\omega}\right|_{\widetilde{F}}=0$ are both equivalent to $F$ being a Lagrangian subvariety of $X$.

Proof of (2). By Remark 9 we have $\underline{\widetilde{\omega}}=v^{*} \underline{\omega}$ and therefore

$$
\left.\widetilde{\omega}\right|_{\widetilde{F}}=\widetilde{i}^{*} v^{*} \underline{\omega}=\left.v\right|_{\widetilde{F}} ^{*} i^{*} \underline{\omega} \in \mathrm{H}^{2}(\widetilde{F}, \mathbb{C}) \text {. }
$$

Hence the condition $i^{*} \underline{\omega}=0$ implies $\left.\widetilde{\omega}\right|_{\widetilde{F}}=0$, which, like in part (1), under the given assumptions is equivalent to $F$ being Lagrangian. The converse implication holds if $F$ is a klt variety because then $\left.v\right|_{\widetilde{F}} ^{*}: \mathrm{H}^{2}(F, \mathbb{C}) \rightarrow \mathrm{H}^{2}(\widetilde{F}, \mathbb{C})$ is injective by Theorem 8 .

The following result is a straightforward generalization of [Mat00, Corollary 1$]$.

Proposition 16. Let $f: X \rightarrow B$ be a Lagrangian fibration of a symplectic variety $(X, \omega)$. Then for every fiber $F$ of $f$ the pullback $i^{*} \underline{\omega}$ of the symplectic class vanishes in $\mathrm{H}^{2}(F, \mathbb{C})$, where $i: F \rightarrow X$ denotes the inclusion. Thus also $i^{* *} \underline{\omega}=0 \in \mathrm{H}^{2}\left(F^{\prime}, \mathbb{C}\right)$ for every fiber component $i^{\prime}: F^{\prime} \rightarrow X$ of $F$.

Proof. The Leray spectral sequence for $f$ and the sheaf $\mathcal{O}_{X}$ gives the map $d_{2}: \mathrm{H}^{2}\left(X, \mathcal{O}_{X}\right) \rightarrow \mathrm{H}^{0}\left(B, R^{2} f_{*} \mathcal{O}_{X}\right)$. The class $\underline{\underline{\omega}}$ lies in $\mathrm{H}^{2}\left(X, \mathcal{O}_{X}\right)$. Let $i: F \rightarrow X$ be the fiber over a point $p \in B$ with residue field $\mathbb{C}(p)$. Then the proper base change map $R^{2} f_{*} \mathcal{O}_{X} \otimes_{\mathcal{O}_{X}} \mathbb{C}(p) \stackrel{\sim}{\longrightarrow} \mathrm{H}^{2}\left(F, \mathcal{O}_{F}\right)$ sends $d_{2}(\underline{\bar{\omega}}) \otimes 1$ to $i^{*} \underline{\bar{\omega}} \in \mathrm{H}^{2}\left(F, \mathcal{O}_{F}\right)$.

As the general fiber is Lagrangian, not completely contained in $X_{\text {sing }}$ and has canonical singularities by Lemma 28, the section $d_{2}(\underline{\bar{\omega}})$ vanishes at the general point $p \in B$ by Lemma 15 . Therefore this section is torsion in $R^{2} f_{*} \mathcal{O}_{X}$. However, as $X$ is symplectic, the canonical sheaf of $X$ is trivial, so $\omega_{X} \cong \mathcal{O}_{X}$. Hence the sheaf $R^{2} f_{*} \mathcal{O}_{X}$ is torsion-free by Kollár's torsion-freeness Theorem, which also holds in the singular case, see Theorem 29 in the appendix. Thus $d_{2}(\underline{\underline{\omega}})=0$, so for every fiber $i: F \rightarrow X$ the class $i^{*} \underline{\omega}$ vanishes. Moreover $i^{\prime *} \underline{\omega}=0$ because $i^{\prime}$ factors through $i$.

Theorem 17. Let $f: X \rightarrow B$ be a Lagrangian fibration of a symplectic variety $(X, \omega)$. Then every component of each fiber of $f$ does not lie completely in $X_{\text {sing }}$ and is Lagrangian. In particular $f$ is equidimensional.

\footnotetext{
${ }^{5} \mathrm{~A}$ resolution of singularities $v: \widetilde{X} \rightarrow X$ that is an isomorphism over $X_{\text {reg }}$ and for which the strict transform $\overline{v^{-1}\left(F \cap X_{\text {reg }}\right)}$ of $F$ is smooth
} 
Proof. We prove this theorem by induction over the dimension of $X$. If $X$ is a surface, all fibers of $f$ are curves. As $X$ is normal, it has only isolated singularities and no fiber component can lie in $X_{\text {sing }}$. By Proposition 16 the symplectic class vanishes on every fiber component $F^{\prime}$. Hence $F^{\prime}$ is a Lagrangian subvariety of $X$ by Lemma 15 and the theorem is proven for $\operatorname{dim} X=2$.

We assume now $2 n:=\operatorname{dim} X>2$ and that the theorem is already proven for all lower dimensional symplectic varieties. Let $i^{\prime}: F^{\prime} \hookrightarrow X$ be a component of a fiber $F$ of $f$. Let $Z$ be an irreducible component

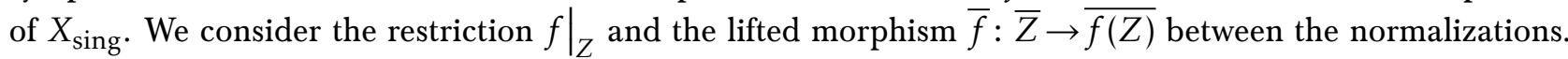
Then by [Mat15, Theorem 3.1] the normalization $\bar{Z}$ is a symplectic variety and $\bar{f}$ is a Lagrangian fibration. By the induction hypothesis, we know that $\bar{f}$ is equidimensional, so every fiber of $\bar{f}$ has dimension at most $n-1$. Hence the dimension of all fibers of $\left.f\right|_{Z}$ is also at most $n-1$. However, by the fiber dimension theorem, [Sha94, Theorem 1.25], we have $\operatorname{dim} F^{\prime} \geq n$, so $F^{\prime} \not \subset Z$ and $F^{\prime}$ cannot lie completely in $X_{\text {sing. Now }}$ Proposition 16 implies $i^{\prime *} \underline{\omega}=0$, so $F^{\prime}$ is by Lemma 15 an $n$-dimensional Lagrangian subvariety of $X$. This completes the induction.

\section{Generalized Beauville-Bogomolov form}

Namikawa defined a generalized Beauville-Bogomolov form on every Namikawa symplectic variety $X$ by pulling back everything to a resolution of singularities $\widetilde{X}$ and then calculating Beauville's formula [Bea83b, p. 772]. We prefer working with Kirschner's idea to calculate all integrals directly on the singular cohomology of $X$, which makes explicit calculations easier. Both approaches are equivalent, compatible with pullbacks and, after a suitable normalizing, define for every irreducible symplectic variety a uniquely determined quadratic form $q_{X}$ on $\mathrm{H}^{2}(X, \mathbb{C})$.

\subsection{Definitions of the Beauville-Bogomolov form}

Definition 18 (Beauville-Bogomolov form, cf. [Nam01c, Theorem 8 (2)]). Let $(X, \omega)$ be a 2n-dimensional, irreducible symplectic variety with a resolution $v: \widetilde{X} \rightarrow X$ and extension $\widetilde{\omega} \in \mathrm{H}^{0}\left(\widetilde{X}, \Omega_{\widetilde{X}}^{2}\right)$ of $v^{*} \omega$ to $\widetilde{X}$. We consider the pullbacks $v^{*} \alpha=: \widetilde{\alpha}$ of classes $\alpha \in \mathrm{H}^{2}(X, \mathbb{C})$ as the classes of two-forms. Then the Beauville-Bogomolov form on $X$ is the quadratic form $q_{X, \omega}: \mathrm{H}^{2}(X, \mathbb{C}) \rightarrow \mathbb{C}$ with

$$
q_{X, \omega}(\alpha):=\frac{n}{2} \int_{\widetilde{X}}(\widetilde{\omega} \overline{\widetilde{\omega}})^{n-1} \widetilde{\alpha}^{2}+(1-n)\left(\int_{\widetilde{X}} \widetilde{\omega}^{n} \overline{\widetilde{\omega}}^{n-1} \widetilde{\alpha}\right) \cdot\left(\int_{\widetilde{X}} \widetilde{\omega}^{n-1} \overline{\widetilde{\omega}}^{n} \widetilde{\alpha}\right)
$$

for all $\alpha \in \mathrm{H}^{2}(X, \mathbb{C})$. The products and powers denote wedge products of forms.

The form $q_{X, \omega}$ does not depend on the chosen resolution. Given two resolutions $v, v^{\prime}$ of $X$, this can be seen by going over to a common resolution factoring through $v$ and $v^{\prime}$.

A resolution $\widetilde{X}$ does not need to be symplectic again. Note that thus we cannot trivially deduce all properties of $q_{X, \omega}$ from the smooth case by considering $\widetilde{X}$.

Definition 19 (Beauville-Bogomolov form, cf. [Kir15, Notation 3.2.1.]). Let $X$ be a $2 n$-dimensional, complex projective variety. We define for every class $w \in \mathrm{H}^{2}(X, \mathbb{C})$ a quadratic form $q_{X, w}: \mathrm{H}^{2}(X, \mathbb{C}) \rightarrow \mathbb{C}$ with

$$
q_{X, w}(v):=\frac{n}{2} \int_{X}(w \bar{w})^{n-1} v^{2}+(1-n)\left(\int_{X} w^{n} \bar{w}^{n-1} v\right) \cdot\left(\int_{X} w^{n-1} \bar{w}^{n} v\right)
$$

for all $v \in \mathrm{H}^{2}(X, \mathbb{C})$. Here the products and powers denote the cup product in the cohomology ring $\mathrm{H}^{*}(X, \mathbb{C})$. Using our notation from Remark 9, for an irreducible symplectic variety $(X, \omega)$ the quadratic form $q_{X, \underline{\omega}}$ is called Beauville-Bogomolov form on $X$.

In order to obtain a uniquely determined quadratic form $q_{X}$ on an irreducible symplectic variety $X$, it is convenient to normalize the symplectic class. The following Lemma ensures that the definition will be well defined. 
Lemma 20 (Existence and uniqueness of the normalizing). Let $X$ be a $2 n$-dimensional, irreducible symplectic variety $X$. For all $v \in \mathrm{H}^{2}(X, \mathbb{C})$ we denote $I(v):=\int_{X}(v \bar{v})^{n}$. Every symplectic class $\underline{\omega} \in \mathrm{H}^{2}\left(X, \Omega_{X}^{[2]}\right) \backslash\{0\}$ induces $a$ normalized class $w:=I(\underline{\omega})^{-\frac{1}{2 n}} \cdot \underline{\omega}$ with $I(w)=1$. The corresponding Beauville-Bogomolov form $q_{X, w}$ does not depend on the choice of $\underline{\omega}$.

Proof. As $X$ is irreducible symplectic, every symplectic class $\alpha \in \mathrm{H}^{0}\left(X, \Omega_{X}^{[2]}\right) \backslash\{0\}$ on $X$ differs only by a constant factor from the class $\omega$, say $\alpha=c \omega$ for $c \in \mathbb{C}^{\times}$. One computes easily that the normalized classes of $c \omega$ and $\omega$ only differ by a factor $\frac{c}{|c|}$ of absolute value one. We see directly from Definition 19 that this does not affect $q_{X, w}$.

Definition 21 (Normalized Beauville-Bogomolov form). Let $X$ be an irreducible symplectic variety. The normalized Beauville-Bogomolov form is defined as $q_{X}:=q_{X, w}$ for any class $w \in \mathrm{H}^{2,0}(X)$ with $I(w)=1$.

Remark 22 (The symmetric bilinear form). The Beauville-Bogomolov form $q_{X}$ on an irreducible symplectic variety $(X, \omega)$ is induced by a symmetric bilinear form. Like Matsushita we also denote it by $q_{X}$, but with two arguments. We have the usual formula $q_{X}(a, b)=\frac{1}{2}\left(q_{X}(a+b)-q_{X}(a)-q_{X}(b)\right)$ for all $a, b \in \mathrm{H}^{2}(X, \mathbb{C})$.

\subsection{Equivalence of the definitions and behaviour under pullbacks}

To relate Namikawa's and Kirschner's definitions of the Beauville-Bogomolov form, we prove in the following lemma that the Definitions 19 and 21 behave well under pullbacks along birational morphisms.

Lemma 23. Let $\pi: Y \rightarrow X$ be a birational morphism from a normal, complex projective variety $Y$ to a symplectic variety $(X, \omega)$ with an extension $\omega^{\prime} \in \mathrm{H}^{0}\left(Y, \Omega_{Y}^{[2]}\right)$ of $\pi^{*} \omega$. Then $\underline{\omega}$ is normalized if and only if $\underline{\omega^{\prime}}$ is normalized and we have

$$
q_{X, \underline{\omega}}=q_{Y, \underline{\omega}^{\prime}} \circ \pi^{*} \text { and } q_{X}=q_{Y} \circ \pi^{*} .
$$

Proof. We noted in Lemma 7 that the integration of top cohomology classes is compatible with pullbacks under birational morphisms. For every $v, w \in \mathrm{H}^{2}(X, \mathbb{C})$ we can apply this to the occurring integrals in Definition 19 of the Beauville-Bogomolov form, such that we get the relation $q_{X, w}(v)=q_{Y, \pi^{*} w}\left(\pi^{*} v\right)$ for all $v, w \in \mathrm{H}^{2}(X, \mathbb{C})$. Together with $\pi^{*} \underline{\omega}=\underline{\omega}^{\prime} \in \mathrm{H}^{2}(Y, \mathbb{C})$ by Remark 9 this shows the first equality. Similarly we see $I(\underline{\omega})=I\left(\pi^{*} \underline{\omega}\right)=I\left(\underline{\omega^{\prime}}\right)$, which concludes the proof.

Corollary 24 (Equivalence of the definitions, cf. [Kir15, Proposition 3.2.15]). On every irreducible symplectic variety $(X, \omega)$ Namikawa's and Kirschner's definitions of the Beauville-Bogomolov form are equivalent in terms of $q_{X, \omega}=q_{X, \underline{\omega}}$.

Proof. Let $v: \widetilde{X} \rightarrow X$ be a resolution and $\widetilde{\omega}$ the extension of $v^{*} \omega$ to $\widetilde{X}$. Using the notation of Kirschner's Definition 19, we can write Namikawa's Definition 18 as $q_{X, \omega}=q_{\widetilde{X}, \underline{\widetilde{\omega}}} \circ v^{*}$. This equals $q_{X, \underline{\omega}}$ by Lemma 23 .

\section{Proofs of the main results}

\subsection{Fujiki relations and the index of the Beauville-Bogomolov form}

A natural approach to prove Theorem 2 would be to pass to a resolution of singularities, but this might not be a symplectic variety anymore. Instead, we deduce the Fujiki relation from a terminal model, which we have seen to be a Namikawa symplectic variety. Part (2) of Theorem 2 will follow directly from Proposition 25 that contains more information about the decomposition of $\mathrm{H}^{2}(X, \mathbb{C})$. The Hodge theory of $\mathrm{H}^{2}(X, \mathbb{C})$ and the Fujiki relations allow us to calculate the index by hand.

Proof of Theorem 2, part (1). Let $X$ be a $2 n$-dimensional, irreducible symplectic variety with normalized Beauville-Bogomolov form $q_{X}$ on $\mathrm{H}^{2}(X, \mathbb{C})$ as in Definition 21. We need to show that there is a constant $c_{X} \in \mathbb{R}^{+}$, such that for all $v \in \mathrm{H}^{2}(X, \mathbb{C})$ we have the Fujiki relation $c_{X} \cdot q_{X}(v)^{n}=\int_{X} v^{2 n}$.

We write $q_{X}=q_{X, \omega}$ for a normalized symplectic class $\omega$ with $\int_{X}(\underline{\omega \bar{\omega}})^{n}=1$. We take a terminal model $\pi: Y \rightarrow X$ of $X$ together with an extension $\omega^{\prime} \in \mathrm{H}^{2}\left(Y, \Omega_{Y}^{[2]}\right)$ of $\pi^{*} \omega$ from Theorem 5. Then $\left(Y, \omega^{\prime}\right)$ is a 
Namikawa symplectic variety by Proposition 11. We have $q_{Y}=q_{Y, \omega^{\prime}}$ and $q_{X}=q_{Y} \circ \pi^{*}$ by Lemma 23 . Now we apply [Mat01, Theorem 1.2] to $Y$ to get a $c_{Y} \in \mathbb{R}^{+}$, such that for all $u \in \mathrm{H}^{2}(Y, \mathbb{C})$ we get $c_{Y} \cdot q_{Y}(u)^{n}=\int_{Y} u^{2 n}$. Then by the Lemmas 23 and 7 we get for every $v \in \mathrm{H}^{2}(X, \mathbb{C})$ :

$$
c_{Y} \cdot q_{X}(v)^{n}=c_{Y} \cdot q_{Y}\left(\pi^{*} v\right)^{n}=\int_{Y}\left(\pi^{*} v\right)^{2 n}=\int_{Y} \pi^{*}\left(v^{2 n}\right)=\int_{X} v^{2 n}
$$

Hence we have proved the Fujiki relation for the constant $c_{X}:=c_{Y}$.

Proposition 25 (Index of the Beauville Bogomolov form $q_{X}$ ). Let $(X, \omega)$ be a $2 n$-dimensional, irreducible symplectic variety with normalized Beauville-Bogomolov form $q_{X}=q_{X, w}$ for $w \in \mathrm{H}^{2}(X, \mathbb{C})$, and let $a \in \mathrm{H}^{2}(X, \mathbb{R})$ be an ample class.

Restricting $q_{X}$ gives a real quadratic form $\mathrm{H}^{2}(X, \mathbb{R}) \rightarrow \mathbb{R}$ and we get the $q_{X}$-orthogonal decomposition $\mathrm{H}^{2}(X, \mathbb{R})=V_{+} \oplus V_{-}$, where $V_{+}:=\langle w+\bar{w}, i w-i \bar{w}, a\rangle_{\mathbb{R}}$ is a 3-dimensional space on which $q_{X}$ is positive definite and

$$
V_{-}:=a^{\perp} \cap \mathrm{H}^{1,1}(X) \cap \mathrm{H}^{2}(X, \mathbb{R})=\left\{d \in \mathrm{H}^{1,1}(X) \cap \mathrm{H}^{2}(X, \mathbb{R}) \mid q_{X}(d, a)=0\right\}
$$

is a space on which $q_{X}$ is negative definite.

Proof that restricting $q_{X}$ gives a real form. An easy computation for arbitrary $v \in \mathrm{H}^{2}(X, \mathbb{C})$ shows that $q_{X, w}(\bar{v})=\overline{q_{X, w}(v)}$, using that the integrals are compatible with complex conjugation. Hence restricting gives a real quadratic form $\mathrm{H}^{2}(X, \mathbb{R}) \rightarrow \mathbb{R}$.

Proof of $V_{+} \perp V_{-}$and the positivity of $q_{X}$ on $V_{+}\left(c f\right.$. [Mat01, (2.1)]). We can use $\int_{X}(w \bar{w})^{n}=1$ and the formula from Remark 22 to calculate the Beauville-Bogomolov bilinear form $q_{X}$ on the classes $w+\bar{w}, i w-i \bar{w}, a$. The Hodge decomposition from Theorem 8 allows us to calculate the occurring integrals via the usual type considerations, which gives the relations $q_{X}(w+\bar{w})=q_{X}(i w-i \bar{w})=1, q_{X}(w+\bar{w}, i w-i \bar{w})=0$, $q_{X}(w+\bar{w}, v)=q_{X}(i w-i \bar{w}, v)=0$ for all $v \in \mathrm{H}^{1,1}(X)$, and $q_{X}(a)=\frac{n}{2} \int_{X}(w \bar{w})^{n-1} a^{2}$.

Therefore $w+\bar{w}, i w-i \bar{w}$ and $a$ are orthogonal with respect to $q_{X}$. The two subspaces $V_{+}$and $V_{-}$ of $\mathrm{H}^{2}(X, \mathbb{C})$ are likewise orthogonal. The restriction $\left.q_{X}\right|_{V_{+}}$is diagonal with eigenvalues $1,1, q_{X}(a)$. As $w^{n-1} \cup a^{3}=0$ by type considerations, the Hodge-Riemann bilinear relations, Corollary 10, show that $q_{X}(a)=\frac{n}{2} \psi_{X, a}\left(w^{n-1}, w^{n-1}\right)$ is positive and therefore $q_{X}$ is positive definite on $V_{+}$.

Proof that $V_{+} \oplus V_{-}=\mathrm{H}^{2}(X, \mathbb{R})$. The classes $w+\bar{w}, i w-i \bar{w}$ and $a$ are obviously real. On the other hand, as $X$ is irreducible symplectic, $\{w, \bar{w}\}$ is a $\mathbb{C}$-basis of $\mathrm{H}^{2,0}(X) \oplus \mathrm{H}^{0,2}(X)$. Hence every real class $v \in \mathrm{H}^{2}(X, \mathbb{R})$ can be decomposed as $v=\alpha w+v^{1,1}+\beta \bar{w}$ with $\bar{\alpha}=\beta \in \mathbb{C}$ and $v^{1,1}=\overline{v^{1,1}} \in \mathrm{H}^{1,1}(X) \cap \mathrm{H}^{2}(X, \mathbb{R})$. We note $q_{X}\left(v^{1,1}-\lambda a, a\right)=0$ for $\lambda:=\frac{q_{X}\left(v^{1,1}, a\right)}{q_{X}(a)} \in \mathbb{R}$. This gives the decomposition

$$
v=\underbrace{\operatorname{Re}(\alpha) \cdot(w+\bar{w})+\operatorname{Im}(\alpha) \cdot(i w-i \bar{w})+\lambda a}_{\in V_{+}}+\underbrace{v^{1,1}-\lambda a}_{\in V_{-}}
$$

Hence we get $V_{+}+V_{-}=\mathrm{H}^{2}(X, \mathbb{R})$. The directness of this sum decomposition will follow from the negativity of $q_{X}$ on $V_{-}$.

Proof of the negativity of $q_{X}$ on $V_{-} .\left(C f\right.$. [Mat01, (2.4)]) Every class $d$ in the space $V_{-}$is a real class of type $(1,1)$ with $q_{X}(d, a)=0$. We need to show $q_{X}(d)<0$ if $d \neq 0$. We use the Fujiki relations on $X$ to calculate for every $t \in \mathbb{R}$ :

$$
\int_{X}(t d+a)^{2 n}=c_{X} \cdot q_{X}(t d+a)^{n}=c_{X} \cdot(t^{2} q_{X}(d)+\underbrace{t q_{X}(d, a)}_{=0}+q_{X}(a))^{n} .
$$

Comparing the $t$ and $t^{2}$-terms on both sides yields $d \cup a^{2 n-1}=0$ and

$$
(2 n-1) \cdot \int_{X}\left(d^{2} \cup a^{2 n-2}\right)=c_{X} \cdot q_{X}(d) \cdot q_{X}(a)^{n-1} .
$$


We have $d=\bar{d}$ because $d$ is real. Therefore the Hodge-Riemann bilinear relations, Corollary 10, imply $\int_{X}\left(d^{2} \cup a^{2 n-2}\right)=\psi_{X, a}(d, d)<0$ and hence $q(d)<0$.

\subsection{Fibrations of irreducible symplectic varieties}

Let $(X, \omega)$ be an irreducible symplectic variety of complex dimension $2 n$, together with a surjective morphism $f: X \rightarrow B$ with connected fibers onto a normal, complex projective variety $B$ with dimension $0<\operatorname{dim} B<2 n$. We subdivide the proof of Theorem 3 into seven steps, which we will prove in the following order:

(1a) $\operatorname{dim} B=n$.

(4a) The general fiber is a Lagrangian subvariety of $X$.

(4b) Every fiber component of $f$ is an $n$-dimensional Lagrangian subvariety of $X$ and does not lie completely in $X_{\text {sing }}$.

(3a) The general fiber is smooth.

(3b) The general fiber is an Abelian variety.

(2) $X$ is smooth along the general fiber and $f\left(X_{\text {sing }}\right) \subset B$ is a proper closed subset.

(lb) $B$ is a $\mathbb{Q}$-factorial klt variety with $\rho(B)=1$.

Proof of Theorem 3. For the proof we choose very ample divisors $A$ on $X$ and $H$ on $B$. We denote their first Chern classes by $a \in \mathrm{H}^{2}(X, \mathbb{C})$ and $h \in \mathrm{H}^{2}(B, \mathbb{C})$. For every class $d=c_{1}\left(\mathcal{O}_{B}(D)\right)$ that comes from a Cartier divisor $D$ on $B$ we get $\left(f^{*} D\right)^{2 n}=0$. Moreover, the Fujiki relation $\left(f^{*} D\right)^{2 n}=c_{X} \cdot q_{X}\left(f^{*} d\right)^{n}$ gives

$$
q_{X}\left(f^{*} d\right)=0 .
$$

Proof of (1a). (Cf. [Mat01, (3.2)]) We calculate for every $t \in \mathbb{R}^{+}$

$$
\left(A+t f^{*} H\right)^{2 n}=c_{X} \cdot q_{X}\left(a+t f^{*} h\right)^{n}=c_{X} \cdot\left(q_{X}(a)+2 t q_{X}\left(a, f^{*} h\right)\right)^{n}
$$

by Equation (1). We see from the $t$-coefficient that $q_{X}\left(a, f^{*} h\right)$ is positive because $A$ is ample:

$$
q_{X}\left(a, f^{*} h\right)=\frac{A^{2 n-1} \cdot f^{*} H}{c_{X} \cdot q_{X}(a)^{n-1}}>0 .
$$

Therefore the $t^{k}$-coefficient $\left(\begin{array}{c}2 n \\ k\end{array}\right) \cdot A^{2 n-k} \cdot\left(f^{*} H\right)^{k}$ vanishes for $k>n$, but survives for $k=n$ :

$$
\left(\begin{array}{c}
2 n \\
n
\end{array}\right) \cdot A^{n} \cdot\left(f^{*} H\right)^{n}=2^{n} c_{X} \cdot q_{X}\left(a, f^{*} h\right)^{n}>0 .
$$

This shows $\operatorname{dim} B=n$.

Proof of (4a). (Cf. [Mat01, (3.3)]) We consider a general fiber $F$ over a smooth point $b \in B$ and the embedding $i: F \hookrightarrow X$. By Lemma 28 in the appendix the general fiber $F$ fulfills $F_{\text {reg }}=F \cap X_{\text {reg }}$ and has at most canonical singularities. Therefore, considering the symplectic form $\omega$ as a holomorphic form on the smooth locus

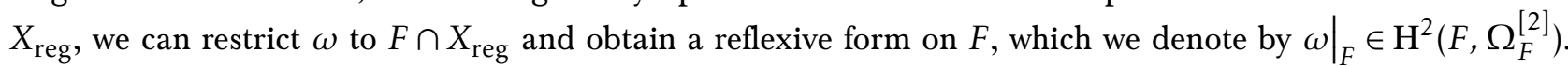
By Lemma 15 we need to prove $\left.\omega\right|_{F}=0$ to show that $F$ is a Lagrangian subvariety of $X$.

We note $\underline{\left.\omega\right|_{F}}=i^{*} w$ for $w:=\underline{\omega}$ and that $i^{*} a$ is an ample class on $F$. The idea is now to show

$$
\int_{F} i^{*}\left(w \bar{w} a^{n-2}\right)=0
$$

This integral equals $\psi_{F, i^{*} a}\left(i^{*} w, i^{*} w\right)$ and by the Hodge-Riemann bilinear relations, Corollary 10, it vanishes if and only if $\left.\omega\right|_{F}=0$, otherwise it is positive.

As $X, F, B$ are compact, complex varieties, they have canonical fundamental classes $[X] \in \mathrm{H}_{4 n}(X, \mathbb{Z})$, $[F] \in \mathrm{H}_{2 n}(F, \mathbb{Z}),[B] \in \mathrm{H}_{2 n}(B, \mathbb{Z})$. By definition, we have $H^{n}=[B] \cap h^{n}$, so the class $[B]^{\vee}:=\frac{1}{H^{n}} h^{n} \in \mathrm{H}^{2 n}(B, \mathbb{Z})$ is dual to $[B]$. 
If $X, F, B$ are smooth, Poincaré duality gives the equality

$$
[X] \cap f^{*}\left([B]^{\vee}\right)=i_{*}[F]
$$

in $\mathrm{H}^{2 n}(X, \mathbb{Z})$. Going over to a commutative diagram of resolutions and doing calculations with the projection formula shows that equation (4) holds also in the singular case, [Sch17, Lemma 63]. We use this to calculate the integral (3).

$$
\begin{array}{rlr}
\int_{F} i^{*}\left(w \bar{w} a^{n-2}\right) & =[F] \cap i^{*}\left(w \bar{w} a^{n-2}\right) & \\
& =i_{*}[F] \cap w \bar{w} a^{n-2} & \text { Projection formula } \\
& =\frac{1}{H^{n}}\left([X] \cap\left(f^{*} h\right)^{n}\right) \cap w \bar{w} a^{n-2} & \text { By equation (4) } \\
& =\frac{1}{H^{n}}[X] \cap\left(w \bar{w} a^{n-2} \cup\left(f^{*} h\right)^{n}\right) & \text { [Bre97, Theorem VI 5.2.(3)] } \\
& =\frac{1}{H^{n}} \int_{X} w \bar{w} a^{n-2}\left(f^{*} h\right)^{n} &
\end{array}
$$

We calculate the latter integral as a term of the following Fujiki relation for $s, t \in \mathbb{R}^{+}$.

$$
\begin{aligned}
\int_{X}\left(w+\bar{w}+s a+t f^{*} h\right)^{2 n} & =c_{X} \cdot q_{X}\left(w+\bar{w}+s a+t f^{*} h\right)^{n} \\
& =c_{X} \cdot\left(q_{X}(w+\bar{w})+s^{2} q_{X}(a)+2 \operatorname{stq} q_{X}\left(a, f^{*} h\right)\right)^{n}
\end{aligned}
$$

In the last step we used the vanishing of $q_{X}\left(f^{*} h\right)$ by equation (1) and the vanishing of $q_{X}(w+\bar{w}, a)$ and $q_{X}\left(w+\bar{w}, f^{*} h\right)$ that follows directly from the definition of the Beauville-Bogomolov form via type considerations. Comparing the $s^{n-2} t^{n}$-terms gives $\int_{X} w \bar{w} a^{n-2}\left(f^{*} h\right)^{n}=0$ and therefore $\left.\omega\right|_{F}=0$.

Proof of (4b). By part (4a) $f$ is a Lagrangian fibration. Hence part (4b) follows from Theorem 17.

Proof of (3a). Let $\mathcal{I}$ denote the ideal sheaf corresponding to $F$ on $X$ and $\mathcal{N}_{F / X}:=\operatorname{Hom}\left(\mathcal{I} / \mathcal{I}^{2}, \mathcal{O}_{F}\right)$ the normal sheaf of $F$ in $X$. As $F$ is a general fiber, we have $\left.\mathcal{N}_{F / X}\right|_{F_{\text {reg }}} \cong \mathcal{N}_{F_{\text {reg }} / X_{\text {reg }}}$ by Lemma 28. As $F$ is Lagrangian, contracting with the symplectic form gives the well-known isomorphism $\mathcal{N}_{F_{\text {reg }} / X_{\text {reg }}} \cong \Omega_{F_{\text {reg }}}^{1}$.

The differential $d f$ induces a morphism $\left.\mathcal{T}_{X_{\text {reg }}}\right|_{F_{\text {reg }}} \rightarrow T_{b} B \times F_{\text {reg, }}$, where we consider $T_{b} B \times F_{\text {reg }}$ as a free sheaf of rank $n$ on $F_{\text {reg. }}$. The kernel of $d f$ is exactly $\mathcal{T}_{F_{\text {reg }}}$ and $d f$ is surjective because of $\operatorname{dim} B+\operatorname{dim} F=\operatorname{dim} X$. The exact sequence of the tangent complex, [Har77, page 182], gives us $\mathcal{N}_{F_{\text {reg }} / X_{\text {reg }}} \cong \mathcal{T}_{X_{\text {reg }}} \mid F_{\text {reg }} / T_{\text {reg }} \cong T_{b} B \times F_{\text {reg }}$, so the normal bundle $\mathcal{N}_{F_{\text {reg }} / X_{\text {reg }}}$ is also free of rank $n$.

Being the dual of a coherent sheaf, $\mathcal{N}_{F / X}$ is reflexive, [Har80, Corollary 1.2]. As $F$ is normal, the isomorphism $\left.\mathcal{N}_{F / X}\right|_{F_{\text {reg }}} \stackrel{\sim}{\longrightarrow} \Omega_{F_{\text {reg }}}^{1}$ extends uniquely to an isomorphism $\mathcal{N}_{F / X} \stackrel{\sim}{\longrightarrow} \Omega_{F}^{[1]}$ of free sheaves on $F$, [Har80, Proposition 1.6].

Therefore the tangent sheaf $\mathcal{T}_{F}=\left(\Omega_{F}^{[1]}\right)^{*}$ is globally free and $F$ is smooth by the Lipman-Zariski conjecture, which is already proven for the singularities of the minimal model program, [GKKP11, Theorem 6.1], [GK14, Corollary 1.3], [Drul4, Theorem 1.1].

Proof of (3b). As we showed in part (3a) that the general fiber $F$ of $f$ is a compact Kähler manifold with globally free tangent sheaf, we may directly conclude by [Wan54, Corollary 2] that $F$ is an Abelian variety. A more algebraic argument is to use the freeness of $\Omega_{F}^{1}$, so $h^{0}\left(F, \Omega_{F}^{1}\right)=\operatorname{dim} F$. The Kodaira dimension $\mathcal{k}(F)$ is zero because $K_{F}=0$. Then by [Kaw81, Corollary 2] the Albanese morphism $\alpha: F \rightarrow \operatorname{Alb}(F)$ is a birational morphism to the Abelian variety $\mathrm{Alb}(F)$. In fact $\alpha$ is an isomorphism because $K_{F}$ is numerically effective.

Proof of (2). As $X_{\text {sing }}$ is closed and $f$ is proper, $f\left(X_{\text {sing }}\right)$ is closed too. By part (3a) the general fiber $F$ of $f$ is smooth. It is completely contained in $X_{\text {reg }}$ by Lemma 27 in the appendix. Thus $f\left(X_{\text {sing }}\right)$ is a proper closed subset of $B$. 
Proof of (1b). We may assume here that $X$ is Namikawa symplectic, as for the following argument we can go over to a terminal model of $X$. Let $D$ be a Weil divisor on $B$ and $X_{0}:=f^{-1}\left(B_{\text {reg }}\right)$. As $D$ is Cartier on $B_{\text {reg }}$, we can consider the pullback $\left.f\right|_{X_{0}} ^{*}\left(\left.D\right|_{B_{\text {reg }}}\right)$. This induces a divisor $\widetilde{D}$ on $X$ by taking the closures of its components. Then $m \widetilde{D}$ is Cartier for an $m \in \mathbb{N}_{>0}$ because $X$ is $\mathbb{Q}$-factorial, and we get a class $d:=\frac{1}{m} c_{1}\left(\mathcal{O}_{X}(m \widetilde{D})\right)$ in $\mathrm{H}^{2}(X, \mathbb{C})$.

We have $\operatorname{codim}\left(X \backslash X_{0}\right)=\operatorname{codim} B_{\text {sing }} \geq 2$ because $f$ is equidimensional by part $(4 \mathrm{~b})$ and $B$ is normal. Hence no component of $\widetilde{D}$ or $D$ lies outside of $X_{0}$ or $B_{\text {reg }}$, respectively. This implies $f_{*} \widetilde{D}=D$ as Weil divisors and if $D$ is Cartier also $\widetilde{D}=f^{*} D$ and $d=f^{*} c_{1}\left(\mathcal{O}_{B}(D)\right)$.

Like in [Mat99, Step 4], we calculate now for $t \in \mathbb{R}$ the Fujiki relation

$$
\begin{aligned}
(\widetilde{D} & \left.+t f^{*} H+s A\right)^{2 n} \\
& =c_{X} \cdot\left(q_{X}(d)+s^{2} q_{X}(a)+2 t q_{X}\left(d, f^{*} h\right)+2 s q_{X}(d, a)+2 s t q_{X}\left(f^{*} h, a\right)\right)^{n},
\end{aligned}
$$

where we used $q_{X}\left(f^{*} h\right)=0$, and compare coefficients. Considering the constant term, of $t^{n}$ and of $t^{n-1} s^{n-1}$ gives constants $c_{1}, \ldots, c_{4} \in \mathbb{Q}^{+}$with

$$
\begin{aligned}
\widetilde{D}^{2 n} & =c_{1} \cdot q_{X}(d)^{n} \\
\widetilde{D}^{n} \cdot\left(f^{*} H\right)^{n} & =c_{2} \cdot q_{X}\left(d, f^{*} h\right)^{n} \\
\widetilde{D}^{2} \cdot\left(f^{*} H\right)^{n-1} \cdot A^{n-1} & =c_{3} \cdot q_{X}(d) q_{X}\left(f^{*} h, a\right)^{n-1} \\
& +c_{4} \cdot q_{X}\left(d, f^{*} h\right) q_{X}(d, a) q_{X}\left(f^{*} h, a\right)^{n-2} .
\end{aligned}
$$

We show that all three intersection products vanish. For this we may substitute the factors $H$ by general hyperplane sections $H_{i}$ of $B$. By Bertini's Theorem $H_{1}, \ldots, H_{n-1}$ intersect in a smooth curve $C \subset B_{\text {reg }}$ that meets $D$ transversally in finitely many points in $B_{\text {reg }}$, [Aki51]. Thus the intersection products $\widetilde{D}^{n} \cdot\left(f^{*} H\right)^{n}$ and $\widetilde{D}^{2} \cdot\left(f^{*} H\right)^{n-1} \cdot A^{n-1}$ can be calculated over $B_{\text {reg. }}$. As $X$ is Cohen Macaulay and $f$ equidimensional, the restriction $\left.f\right|_{X_{0}}$ is flat, [Eis95, Theorem 18.16]. By [Ful98, Proposition 2.5d] we can calculate

$$
\begin{gathered}
\widetilde{D}^{n} \cdot\left(f^{*} H\right)^{n}=f^{*}\left(\left.\left.D\right|_{B_{\text {reg }}} ^{n} \cdot H\right|_{B_{\text {reg }}} ^{n}\right)=0, \\
\widetilde{D}^{2} \cdot\left(f^{*} H\right)^{n-1} \cdot A^{n-1}=\left.f^{*}\left(\left.\left.D\right|_{B_{\text {reg }}} ^{2} \cdot H\right|_{B_{\text {reg }}} ^{n-1}\right) \cdot A\right|_{X_{0}} ^{n-1}=0
\end{gathered}
$$

because $\operatorname{dim} B=n$. Hence $q_{X}\left(d, f^{*} h\right)=0$, and due to $q_{X}\left(f^{*} h, a\right)>0$ also $q_{X}(d)=0$, which gives $\widetilde{D}^{2 n}=0$.

The vanishing of $q_{X}\left(d, f^{*} h\right), q_{X}(d), q_{X}\left(f^{*} h\right)$ give the Fujiki relation $\left(\widetilde{D}-\lambda f^{*} H\right)^{2 n}=c_{X} \cdot q_{X}\left(d-\lambda f^{*} h\right)^{n}=0$ for every $\lambda \in \mathbb{R}$. For $\lambda:=\frac{\widetilde{D} \cdot A^{2 n-1}}{f^{*} H \cdot A^{2 n-1}} \in \mathbb{Q}$ we get also $\left(\widetilde{D}-\lambda f^{*} H\right) \cdot A^{2 n-1}=0$, thus the $t^{2 n-1}$ terms of the Fujiki relation

$$
\left(\widetilde{D}-\lambda f^{*} H+t A\right)^{2 n}=c_{X} \cdot\left(2 t q_{X}\left(d-\lambda f^{*} h, a\right)+t^{2} q_{X}(a)\right)^{n}
$$

vanish. So $q_{X}\left(d-\lambda f^{*} h, a\right)=0$ and therefore $d-\lambda f^{*} h \in a^{\perp} \cap \mathrm{H}^{1,1}(X) \cap \mathrm{H}^{2}(X, \mathbb{R})$. The form $q_{X}$ is by Proposition 25 negative definite on this space, so $d=\lambda f^{*} h$ in $\mathrm{H}^{2}(X, \mathbb{Q})$ and $\widetilde{D} \equiv \lambda f^{*} H$.

Now, if like in Matsushita's setting $D$ is $\mathbb{Q}$-Cartier, we get $D \equiv \lambda H$ by the projection formula, so $\rho(B)=1$. In the general case we show that $D$ is numerically $\mathbb{Q}$-Cartier in the sense of [HMP15]. This means that we have to construct for any resolution $\mu: \widetilde{B} \rightarrow B$ a $\mu$-trivial $\mathbb{Q}$-Cartier divisor $D^{\prime}$ on $\widetilde{B}$ with $\mu_{*} D^{\prime}=D$.

For every resolution $\mu: \widetilde{B} \rightarrow B$ the fiber product $X \times{ }_{B} \widetilde{B}$ consists of the exceptional components of $\mu$ and a component $Y$ that is isomorphic to the graph of $f$ and lies birational over $X$. Hence we can construct a commutative diagram of resolutions

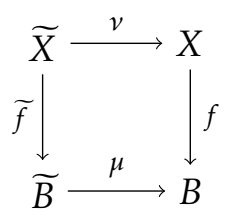


by taking $\widetilde{X}$ to be a resolution of $Y$, together with the morphisms $v, \widetilde{f}$ induced by the projections from the fiber product onto $X, B$. We consider $D^{\prime}:=\widetilde{f} v^{*} \widetilde{D}$. As $v^{*} \widetilde{D} \equiv \lambda v^{*} f^{*} H=\lambda \widetilde{f}^{*} \mu^{*} H$, we have $D^{\prime} \equiv \lambda \mu^{*} H$, which is $\mu$-trivial with $\mu_{*} D^{\prime}=\mu_{*} \widetilde{f}_{*} v^{*} \widetilde{D}=f_{*} v_{*} v^{*} \widetilde{D}=D$, so $D$ is numerically Q-Cartier.

Fujino's result [Fuj99, Theorem 1.2] shows that $B$ is a klt variety, so it has rational singularities. Hence by [HMP15, Theorem 5.11] $D$ is $\mathbb{Q}$-Cartier, so $B$ is $\mathbb{Q}$-factorial.

\subsection{Fibrations of primitive symplectic varieties}

We give an example showing that part 1 of Theorem 4 can fail for an irreducible symplectic variety. The idea is due to Matsushita and was worked out by Sawon [Mat01, p. 7f], [Saw14, Lemma 15].

Example 26. Consider the elliptic curve $E=\mathbb{C} / \Gamma$ with the lattice $\Gamma=\mathbb{Z}+\mathbb{Z} \cdot \zeta_{6}$, where $\zeta_{6}$ is a primitive sixth root of unity, and the 6-torus $T:=E^{6}$. The maps

$$
\begin{aligned}
\mathbb{C}^{6} & \rightarrow \mathbb{C}^{6} \\
\left(z_{1}, \ldots, z_{6}\right) & \mapsto\left(\zeta_{6} z_{1}, \zeta_{6}^{5} z_{2},-z_{3},-z_{4}, \zeta_{6}^{2} z_{5}, \zeta_{6}^{4} z_{6}\right) \\
\left(z_{1}, \ldots, z_{6}\right) & \mapsto\left(z_{5}, z_{6}, z_{1}, z_{2}, z_{3}, z_{4}\right)
\end{aligned}
$$

generate a group of automorphisms of $\mathbb{C}^{6}$ preserving $\Gamma^{6} \subset \mathbb{C}^{6}$, which induces a subgroup $G \subset$ Aut $T$. Then $X:=T / G$ is an irreducible symplectic variety with $\omega:=d z_{1} \wedge d z_{2}+d z_{3} \wedge d z_{4}+d z_{5} \wedge d z_{6}$, [Bea00, Proposition 2.4]. However, it is not primitive symplectic, as it carries holomorphic 3-forms. The group $G$ also acts on the subtorus $T^{\prime}$ with coordinates $\left(z_{1}, z_{3}, z_{5}\right)$ and the quotient $B:=T^{\prime} / G$ carries the holomorphic form $d z_{1} \wedge d z_{3} \wedge d z_{5}$, hence $K_{B}=0$, and B has canonical singularities due to the Reid-Tai criterion [Kol13, Theorem 3.21].

Proof of Theorem 4. Let $(X, \omega)$ be a primitive symplectic variety with a morphism $f: X \rightarrow B$ like in Theorem 4 . We have to show the following two parts.

(1) The base variety $B$ is Fano.

(2) If $B$ is smooth, then $B \cong \mathbb{P}^{n}$.

Proof of (1). As $B$ is $\mathbb{Q}$-factorial with $\rho(B)=1$ by Theorem 3, the canonical divisor $K_{B}$ is $\mathbb{Q}$-Cartier with $K_{B} \equiv t H$ for a $t \in \mathbb{Q}$. By the Iitaka conjecture, which due to Kawamata is known to hold in this special case, see Theorem 30 in the appendix, we have $\kappa(B) \leq 0$, so $t \leq 0$. Therefore we only need to exclude the case $t=0$. Up to here this is the same idea as in [Mat99, step 5].

We assume that $K_{B}$ is numerically trivial. Applying [Kaw85, Theorem 8.2] to a terminal model of $B$ shows that there is a $d \in \mathbb{N}_{>0}$, such that $\mathcal{O}_{B}\left(d K_{B}\right) \cong \mathcal{O}_{B}$ is trivial. As the canonical sheaf is a reflexive sheaf that is locally free on $X_{\text {reg }}$, we can go over to a ramified cyclic covering like in [KM98, 2.52-2.53]. This is a finite morphism $p: \hat{B} \rightarrow B$ with $p^{*} \mathcal{O}_{B}\left(K_{B}\right)=\mathcal{O}_{\hat{B}}$ that is étale of degree $d$ over $X_{\text {reg }}$ and possibly branched over $X_{\text {sing. Hence }} p$ is quasi-étale because $X$ is normal. The variety $\hat{B}$ is by [KM98, Proposition 5.20] also a klt variety. We consider the fiber product $X \times_{B} \hat{B}$ and take the normalization $\hat{X}$ of any component that

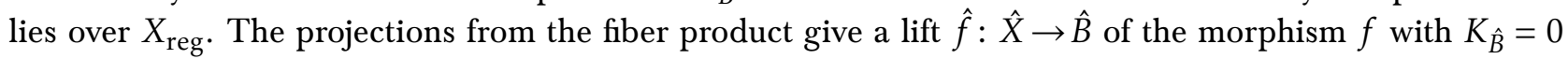
and a quasi-étale morphism $\pi: \hat{X} \rightarrow X$. There is a non-zero reflexive form $\alpha \in \mathrm{H}^{0}\left(\hat{B}, \Omega_{\hat{B}}^{[n]}\right)$ and, as $\hat{B}$ is a klt variety, we can pull it back by Theorem 5 to a non-zero reflexive form $\hat{f}^{*} \alpha \in \mathrm{H}^{0}\left(\hat{X}, \Omega_{\hat{X}}^{[n]}\right)$. As $\hat{f}^{*} \alpha$ is not zero, $n$ has to be even and $\hat{f}^{*} \alpha$ a multiple of $\pi^{*}(\omega)^{n / 2}$ because $X$ is primitive symplectic. This is a contradiction because $\hat{f}^{*} \alpha^{2}=0$, but $\pi^{*} \omega^{n}$ has no zeros.

Proof of (2). As $X$ is primitive symplectic, every terminal model $\pi: Y \rightarrow X$ of $X$ is also primitive symplectic by Proposition 12. Then $\mathrm{h}^{p}\left(Y, \mathcal{O}_{Y}\right)=\mathrm{h}^{0}\left(Y, \Omega_{X}^{[p]}\right)$ for all $p$ by Hodge symmetry, [GKP16, Proposition 6.9], so $Y$ is cohomologically irreducible symplectic in Matsushita's terminology, [Mat15, Definition 1.6]. Applying Matsushita's result [Mat15, Theorem 1.10] to $f \circ \pi: Y \rightarrow B$ shows $B \cong \mathbb{P}^{n}$ if $B$ is smooth. 


\section{Appendix}

We explain here some probably well-known results used in the main proofs above. Lemma 27 can be seen as a special case of [Kol96, Theorem I.6.5]. Theorems 29 and 30 are easy but useful consequences of results of Kollár and Kawamata.

Lemma 27 (Smoothness at general fibers). Let $f: X \rightarrow Y$ be a surjective morphism of complex varieties with a general fiber $F$. If $F$ is smooth at a point $x \in F$, then $X$ is smooth at $x$.

Proof. Let $X, Y$ be $n+r$ and $n$-dimensional, respectively. A general fiber $F$ lies over a smooth point $y \in Y$ and has the dimension $r$ by the fiber dimension theorem. Consider a smooth point $x \in F$ with maximal ideals $\mathfrak{m}_{X, x} \subset \mathcal{O}_{X, x}$ and $\mathfrak{m}_{F, x} \subset \mathcal{O}_{F, x}$ and the maximal ideal $\mathfrak{m}_{Y, y} \subset \mathcal{O}_{Y, y}$ of $y$. As $y \in Y$ and $x \in F$ are regular, we can write $\mathfrak{m}_{F, x}=\left(\overline{\phi_{1}}, \ldots, \overline{\phi_{r}}\right)$ and $\mathfrak{m}_{Y, y}=\left(\psi_{1}, \ldots, \psi_{n}\right)$. The ideal sheaf sequence for $F \subset X$ allows us to extend the $\overline{\phi_{i}}$ to functions $\phi_{i} \in \mathfrak{m}_{X, x} \subset \mathcal{O}_{X, x}$. Then it can easily be checked that we get a short exact sequence

$$
0 \rightarrow f^{*} \mathfrak{m}_{Y, y} \rightarrow \mathfrak{m}_{X, x} \rightarrow \mathfrak{m}_{F, x} \rightarrow 0
$$

that shows $\mathfrak{m}_{X, x}=\left(f^{*} \psi_{1}, \ldots, f^{*} \psi_{n}, \phi_{1}, \ldots, \phi_{r}\right)$. Hence $x \in X$ is regular and also smooth because we work over a perfect field.

Lemma 28 (Singularities of general fibers). Let $f: X \rightarrow Y$ be a surjective morphism of complex varieties with connected fibers, where $X$ is normal. Then the general fiber $F$ is irreducible with $F_{\text {sing }}=F \cap X_{\text {sing. }}$. When $X$ has canonical (resp. terminal) singularities, the general fiber also has canonical (resp.terminal) singularities.

Proof. A general fiber $F$ lies over a smooth point $y \in Y$. Let $L_{y}$ denote the linear system of hyperplanes passing through $y$. Then the fiber $F$ is the base locus of the linear system $f^{*} L_{y}$. The general member of $f^{*} L_{y}$ is normal with singularities contained in $X_{\text {sing }}$ [BS95, Theorem 1.7.1.1]. It follows by induction over the codimension of $F$ that $F$ is normal with $F_{\text {sing }} \subset F \cap X_{\text {sing. }}$. On the other hand, a smooth point of $F$ is by Lemma 27 also a smooth point of $X$. This gives the equality $F_{\text {sing }}=F \cap X_{\text {sing. }}$. As $F$ is normal and connected, it is also irreducible.

When $v: \widetilde{X} \rightarrow X$ is a resolution, the same argument shows that the strict transform $\widetilde{F}$ of $F$ is smooth. The adjunction formula allows to compare the minimal discrepancies as $\operatorname{discr}(F) \geq \operatorname{discr}(X)$, which shows that $F$ also has canonical (resp. terminal) singularities if $X$ does.

Theorem 29 (Kollár's torsion-freeness). Let $f: X \rightarrow B$ be a surjective morphism with connected fibers between complex projective varieties, where $X$ has rational singularities. Then all higher direct image sheaves $R^{i} f_{*} \omega_{X}$ for $i \geq 0$ are torsion-free sheaves on $B$.

Proof. Let $v: \widetilde{X} \rightarrow X$ be a resolution of singularities. We consider the Grothendieck spectral sequence for the functors $v_{*}, f_{*}$ and the canonical sheaf $\omega_{\widetilde{X}}=\Omega_{\widetilde{X}}^{n}$.

$$
E_{2}^{p q}=R^{p} f_{*}\left(R^{q} v_{*} \omega_{\widetilde{X}}\right) \Rightarrow R^{p+q}(f \circ v)_{*} \omega_{\widetilde{X}}
$$

By Grauert-Riemenschneider vanishing all higher direct images $R^{q} v_{*} \omega_{\widetilde{X}}$ vanish for $q>0$, [Laz04, Theorem 4.3.9]. Hence we are only left with the row $E_{2}^{p 0}$ and the spectral sequence degenerates already on $E_{2}$. As $X$ has rational singularities, we have $v_{*} \omega_{\widetilde{X}}=\omega_{X}$ by [KM98, Lemma 5.12]. This gives us

$$
R^{p} f_{*} \omega_{X} \cong R^{p} f_{*}\left(v_{*} \omega_{\widetilde{X}}\right) \cong R^{p}(f \circ v)_{*} \omega_{\widetilde{X}} .
$$

The latter sheaf is torsion-free by Kollár's result [Kol86, Theorem 2.1].

Theorem 30 (Reduction of the Iitaka conjecture to the minimal model program). Let $f: X \rightarrow B$ be $a$ surjective morphism with connected fibers between normal, complex projective varieties. If the general fiber $F$ has a good minimal model, in other words $F$ is birational to a variety with semiample canonical divisor, then $\kappa(X) \geq \kappa(F)+\kappa(B)$. 
Proof. We construct a commutative diagram like diagram 5 that lifts $f$ to a morphism $\widetilde{f}$ between resolutions $\widetilde{X}, \widetilde{B}$ of $X, B$. The fibers of $\widetilde{f}$ are connected. The general fiber of $\widetilde{f}$ is smooth by Lemma 28 and lies over the general fiber of $f$. As the Kodaira dimension is a birational invariant, Theorem 30 follows now from the smooth case that was proved by Kawamata, [Kaw85, Corollary 1.2].

\section{References}

[Aki51] Yasuo Akizuki, Theorems of Bertini on linear systems, J. Math. Soc. Japan 3 (1951), 170-180, DOI:10.2969/jmsj/00310170.

[BCHM10] Caucher Birkar, Paolo Cascini, Christopher D. Hacon, and James McKernan, Existence of minimal models for varieties of log general type, Journal of the AMS 23 (2010), 405-468, DOI:10.1090/S08940347-09-00649-3.

[Bea83a] Arnaud Beauville, Some remarks on Kähler manifolds with $c_{1}=0$, Classification of algebraic and analytic manifolds (Katata, 1982), Progr. Math., vol. 39, Birkhäuser Boston, Boston, MA, 1983, pp. 1-26.

[Bea83b] Arnaud Beauville, Variétés Kähleriennes dont la première classe de Chern est nulle, J. Differential Geom. 18 (1983), no. 4, 755-782 (1984), Projecteuclid:1214438181.

[Bea00] Arnaud Beauville, Symplectic singularities, Invent. Math. 139 (2000), no. 3, 541-549, DOI:10.1007/s002229900043.

[Bre97] Glen E. Bredon, Topology and geometry, Graduate Texts in Mathematics, vol. 139, Springer-Verlag, New York, 1997, Corrected third printing of the 1993 original DOI:10.1007/978-1-4757-6848-0.

[BS95] Mauro C. Beltrametti and Andrew J. Sommese, The adjunction theory of complex projective varieties, De Gruyter Expositions in Mathematics, vol. 16, Walter de Gruyter \& Co., Berlin, 1995, DOI:10.1515/9783110871746.

[DG18] Stéphane Druel and Henri Guenancia, A decomposition theorem for smoothable varieties with trivial canonical class, J. Éc. polytech. Math. 5 (2018), 117-147, DOI:10.5802/jep.65.

[Dru14] Stéphane Druel, The Zariski-Lipman conjecture for log canonical spaces, Bull. Lond. Math. Soc. 46 (2014), no. 4, 827-835, DOI:10.1112/blms/bdu040.

[Dru18] Stéphane Druel, A decomposition theorem for singular spaces with trivial canonical class of dimension at most five, Invent. Math. 211 (2018), no. 1, 245-296, DOI:10.1007/s00222-017-0748-y.

[Eis95] David Eisenbud, Commutative algebra with a view toward algebraic geometry, Graduate Texts in Mathematics, vol. 150, Springer-Verlag, New York, 1995, DOI:10.1007/978-1-4612-5350-1.

[Fuj87] Akira Fujiki, On the de Rham cohomology group of a compact Kähler symplectic manifold, Algebraic geometry, Sendai, 1985, Adv. Stud. Pure Math., vol. 10, North-Holland, Amsterdam, 1987, pp. 105165.

[Fuj99] Osamu Fujino, Applications of Kawamata's positivity theorem, Proc. Japan Acad. Ser. A Math. Sci. 75 (1999), no. 6, 75-79, DOI:10.3792/pjaa.75.75.

[Ful98] William Fulton, Intersection theory, second ed., Ergebnisse der Mathematik und ihrer Grenzgebiete. 3. Folge. A Series of Modern Surveys in Mathematics [Results in Mathematics and Related Areas. 3rd Series. A Series of Modern Surveys in Mathematics], vol. 2, Springer-Verlag, Berlin, 1998, DOI:10.1007/978-1-4612-1700-8.

[GGK19] Daniel Greb, Henri Guenancia, and Stefan Kebekus, Klt varieties with trivial canonical class: holonomy, differential forms, and fundamental groups, Geom. Topol. 23 (2019), no. 4, 2051-2124, DOI:10.2140/gt.2019.23.2051.

[GH94] Phillip Griffiths and Joseph Harris, Principles of algebraic geometry, Wiley Classics Library, John Wiley \& Sons Inc., New York, 1994, Reprint of the 1978 original, DOI:10.1002/9781118032527. 
[GK14] Patrick Graf and Sándor J. Kovács, An optimal extension theorem for 1-forms and the Lipman-Zariski conjecture, Doc. Math. 19 (2014), 815-830, www.math.uni-bielefeld.de/documenta/vol-19/27.html.

[GKKP11] Daniel Greb, Stefan Kebekus, Sándor J. Kovács, and Thomas Peternell, Differential forms on log canonical spaces, Inst. Hautes Études Sci. Publ. Math. 114 (2011), no. 1, 87-169, DOI:10.1007/s10240011-0036-0 An extended version with additional graphics is available as arXiv:1003.2913v4.

[GKP16] Daniel Greb, Stefan Kebekus, and Thomas Peternell, Singular spaces with trivial canonical class, Minimal Models and Extremal Rays, Kyoto, 2011 (Tokyo), Adv. Stud. Pure Math., vol. 70, Mathematical Society of Japan, Tokyo, 2016, http://bookstore.ams.org/aspm-70, pp. 67-113.

[GL14] Daniel Greb and Christian Lehn, Base Manifolds for Lagrangian Fibrations on Hyperkähler Manifolds, International Mathematics Research Notices 2014 (2014), no. 19, 5483, DOI:10.1093/imrn/rnt133.

[Har77] Robin Hartshorne, Algebraic geometry, Springer-Verlag, New York, 1977, Graduate Texts in Mathematics, No. 52. DOI:10.1007/978-1-4757-3849-0.

[Har80] Robin Hartshorne, Stable reflexive sheaves, Math. Ann. 254 (1980), no. 2, 121-176, DOI: 10.1007/BF01467074.

[HMP15] Christopher D. Hacon, Mircea Mustaţă, and Mihnea Popa (eds.), Recent advances in algebraic geometry, London Mathematical Society Lecture Note Series, vol. 417, Cambridge University Press, Cambridge, 2015, A volume in honor of Rob Lazarsfeld's 60th birthday, Papers from the conference held at the University of Michigan, Ann Arbor, MI, May 16-19, 2013, DOI:10.1017/CBO9781107416000.

[HNW11] Daniel Huybrechts and Marc Nieper-Wisskirchen, Remarks on derived equivalences of Ricci-flat manifolds, Math. Z. 267 (2011), no. 3-4, 939-963, DOI:10.1007/s00209-009-0655-z.

[HP19] Andreas Höring and Thomas Peternell, Algebraic integrability of foliations with numerically trivial canonical bundle, Inventiones mathematicae (2019), DOI:10.1007/s00222-018-00853-2.

[Hwa08] Jun-Muk Hwang, Base manifolds for fibrations of projective irreducible symplectic manifolds, Inventiones mathematicae 174 (2008), no. 3, 625-644, DOI:10.1007/s00222-008-0143-9.

[HX19] Daniel Huybrechts and Chenyang Xu, Lagrangian fibrations of hyperkähler fourfolds, ArXiv Mathematics e-prints (2019), Preprint: arXiv:1902.10440.

[Kal06] Dmitry Kaledin, Symplectic singularities from the Poisson point of view, J. Reine Angew. Math. 600 (2006), 135-156, DOI:10.1515/CRELLE.2006.089.

[Kaw81] Yujiro Kawamata, Characterization of abelian varieties, Compositio Math. 43 (1981), no. 2, 253-276, www.numdam.org/item?id=CM_1981_43_2_253_0.

[Kaw85] Yujiro Kawamata, Minimal models and the Kodaira dimension of algebraic fiber spaces, J. Reine Angew. Math. 363 (1985), 1-46, DOI:10.1515/crll.1985.363.1.

[Keb13] Stefan Kebekus, Pull-back morphisms for reflexive differential forms, Adv. Math. 245 (2013), 78-112, DOI:10.1016/j.aim.2013.06.013.

[Kir15] Tim Kirschner, Period mappings with applications to symplectic complex spaces, Lecture Notes in Mathematics, vol. 2140, Springer, Cham, 2015, DOI:10.1007/978-3-319-17521-8.

[KM98] János Kollár and Shigefumi Mori, Birational geometry of algebraic varieties, Cambridge Tracts in Mathematics, vol. 134, Cambridge University Press, Cambridge, 1998, DOI:10.1017/CBO9780511662560.

[Kol86] János Kollár, Higher direct images of dualizing sheaves. I, Ann. of Math. (2) 123 (1986), no. 1, 11-42, DOI:10.2307/1971351.

[Kol96] János Kollár, Rational curves on algebraic varieties, Ergebnisse der Mathematik und ihrer Grenzgebiete. 3. Folge. A Series of Modern Surveys in Mathematics [Results in Mathematics and Related Areas. 3rd Series. A Series of Modern Surveys in Mathematics], vol. 32, Springer-Verlag, Berlin, 1996, DOI:10.1007/978-3-662-03276-3. 
[Kol13] János Kollár, Singularities of the minimal model program, Cambridge Tracts in Mathematics, vol. 200, Cambridge University Press, Cambridge, 2013, With a collaboration of Sándor Kovács, DOI:10.1017/CBO9781139547895.

[KP16] Stefan Kebekus and Thomas Peternell, Aspects of the geometry of varieties with canonical singularities., Foliation theory in algebraic geometry. Proceedings of the conference, New York, NY, USA, September 3-7, 2013, Cham: Springer, 2016, DOI:10.1007/978-3-319-24460-0_4, pp. 73-102 (English).

[Laz04] Robert Lazarsfeld, Positivity in algebraic geometry. I, Ergebnisse der Mathematik und ihrer Grenzgebiete. 3. Folge. A Series of Modern Surveys in Mathematics [Results in Mathematics and Related Areas. 3rd Series. A Series of Modern Surveys in Mathematics], vol. 48, Springer-Verlag, Berlin, 2004, Classical setting: line bundles and linear series.

[Mat99] Daisuke Matsushita, On fibre space structures of a projective irreducible symplectic manifold, Topology 38 (1999), no. 1, 79-83, DOI:10.1016/S0040-9383(98)00003-2.

[Mat00] Daisuke Matsushita, Equidimensionality of Lagrangian fibrations on holomorphic symplectic manifolds, Math. Res. Lett. 7 (2000), no. 4, 389-391, DOI:10.4310/MRL.2000.v7.n4.a4.

[Mat01] Daisuke Matsushita, Fujiki relation on symplectic varieties, ArXiv Mathematics e-prints (2001), arXiv:0109165.

[Mat05] Daisuke Matsushita, Higher direct images of dualizing sheaves of Lagrangian fibrations, Amer. J. Math. 127 (2005), no. 2, 243-259, DOI:10.1353/ajm.2005.0009.

[Mat15] Daisuke Matsushita, On base manifolds of Lagrangian fibrations, Sci. China Math. 58 (2015), no. 3, 531-542, DOI:10.1007/s11425-014-4927-7.

[Nam01a] Yoshinori Namikawa, A note on symplectic singularities, ArXiv Mathematics e-prints (2001), arXiv:0101028.

[Nam01b] Yoshinori Namikawa, Deformation theory of singular symplectic n-folds, Math. Ann. 319 (2001), no. 3, 597-623, DOI:10.1007/PL00004451.

[Nam01c] Yoshinori Namikawa, Extension of 2-forms and symplectic varieties, J. Reine Angew. Math. 539 (2001), 123-147, DOI:10.1515/crll.2001.070.

[Nam06] Yoshinori Namikawa, On deformations of $\mathbb{Q}$-factorial symplectic varieties, J. Reine Angew. Math. 599 (2006), 97-110, DOI:10.1515/CRELLE.2006.079.

[Ou16] Wenhao Ou, Lagrangian fibrations on symplectic fourfolds, Journal für die reine und angewandte Mathematik (Crelles Journal) (2016), DOI:10.1515/crelle-2016-0004.

[Saw14] Justin Sawon, Isotrivial elliptic K3 surfaces and Lagrangian fibrations, ArXiv Mathematics e-prints (2014), Preprint: arXiv:1406.1233.

[Sch17] Martin Schwald, Hodge theoretic methods in the study of symplectic varieties, Ph.D. thesis, AlbertLudwigs-Universität Freiburg, May 2017, DOI:10.6094/UNIFR/12695.

[Sha94] Igor R. Shafarevich, Basic algebraic geometry. 1, second ed., Springer-Verlag, Berlin, 1994, Varieties in projective space, Translated from the 1988 Russian edition and with notes by Miles Reid. DOI:10.1007/978-3-642-37956-7.

[Wan54] Hsien-Chung Wang, Complex parallisable manifolds, Proc. Amer. Math. Soc. 5 (1954), 771-776, DOI:10.1090/S0002-9939-1954-0074064-3. 